$$
\begin{aligned}
& \alpha_{0}
\end{aligned}
$$

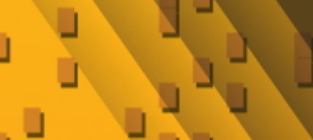

$$
\begin{aligned}
& \text { AVgebra \& } \\
& \text { Number } \\
& \text { Theory }
\end{aligned}
$$

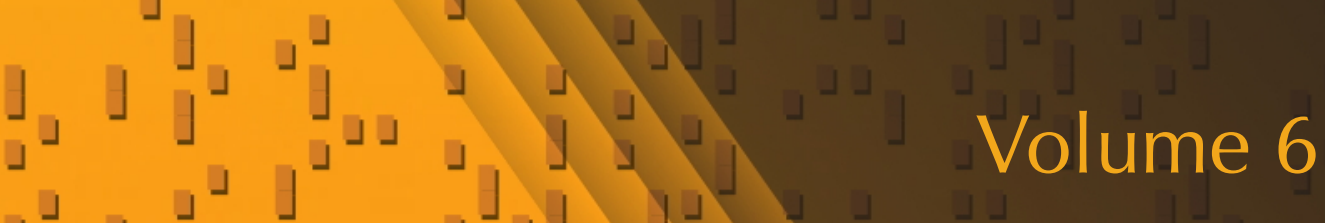

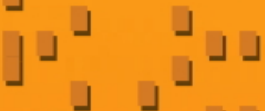

$$
\begin{aligned}
& \lrcorner \\
& \lrcorner \quad\lrcorner\lrcorner
\end{aligned}
$$

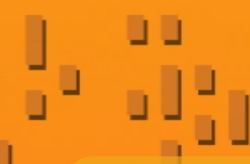

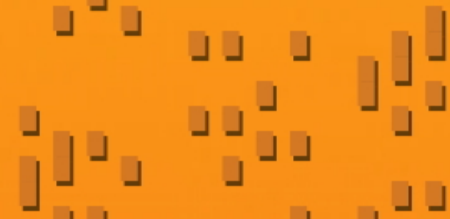

$$
\begin{aligned}
& \lrcorner\lrcorner\lrcorner\lrcorner\lrcorner\lrcorner\lrcorner\lrcorner
\end{aligned}
$$

Arithmetic of singular Enriques surfaces

Klaus Hulek and Matthias Schütt

J.

」

J.

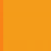




\title{
Arithmetic of singular Enriques surfaces
}

\author{
Klaus Hulek and Matthias Schütt
}

\author{
Dedicated to the memory of Eckart Viehweg
}

\begin{abstract}
We study the arithmetic of Enriques surfaces whose universal covers are singular $\mathrm{K} 3$ surfaces. If a singular K3 surface $X$ has discriminant $d$, then it has a model over the ring class field $H(d)$. Our main theorem is that the same holds true for any Enriques quotient of $X$. It is based on a study of Néron-Severi groups of singular K3 surfaces. We also comment on Galois actions on divisors of Enriques surfaces.
\end{abstract}

\section{Introduction}

Enriques surfaces have formed a vibrant research area over the last 30 years. In many respects, they share the properties of K3 surfaces, yet in other aspects they behave differently. This twofold picture is illustrated in this paper which investigates arithmetic aspects of Enriques surfaces.

The arithmetic of Enriques surfaces is only partially well-understood. For instance, Bogomolov and Tschinkel [1998] proved that potential density of rational points holds on Enriques surfaces. The cited work predates all substantial progress on $\mathrm{K} 3$ surfaces in the same direction. In fact, until now the corresponding statement for K3 surfaces has not been proved in full generality.

In this paper, we investigate the arithmetic of those Enriques surfaces whose universal covers are singular K3 surfaces, i.e., K3 surfaces with Picard number $\rho=20$. We will refer to them as singular Enriques surfaces. Singular K3 surfaces are closely related to elliptic curves with complex multiplication (CM). These structures will be crucial to our investigations; often they explain arithmetic properties of singular K3 surfaces (see Sections 3 and 6).

We point out one particular property that illustrates these relations: the field of definition. A singular K3 surface of discriminant $d$ has a model over the ring class field $H(d)$ just like elliptic curves with $\mathrm{CM}$ in an order of discriminant $d$, by

Partial support from DFG under grant $\mathrm{Hu} 337 / 6-1$ is gratefully acknowledged.

MSC2000: primary 14J28; secondary 11E16, 11G15, 11G35, $14 \mathrm{~J} 27$.

Keywords: Enriques surface, singular K3 surface, elliptic fibration, Néron-Severi group,

Mordell-Weil group, complex multiplication. 
[Schütt 2007, Proposition 4.1]. Our main theorem states how this property carries over to Enriques surfaces:

Theorem 1.1. Let $Y$ be an Enriques surface whose universal cover $X$ is a singular $K 3$ surface. Let $d<0$ denote the discriminant of $X$. Then $Y$ admits a model over the ring class field $H(d)$.

The proof of Theorem 1.1 consists in two steps: first we establish a general result for automorphisms of K3 surfaces over number fields (Proposition 2.1); then we extend the afore-mentioned results for fields of definition of singular K3 surfaces to include their Néron-Severi groups (Theorem 2.4). Here we combine two approaches that both rely on elliptic fibrations. In Section 3 we review the theory of singular K3 surfaces and use Inose's pencil and the theory of Mordell-Weil lattices to deduce Theorem 2.4 for most singular K3 surfaces (see Remark 3.8). On the other hand, Section 4 provides a direct approach for those singular K3 surfaces which are Kummer (Corollary 4.2). Through Shioda-Inose structures, we then connect the two partial results and are thus able to give a full proof of Theorem 2.4 (see 4F).

In Section 5 we address explicit questions. Lattice theoretically one can determine all singular K3 surfaces that admit an Enriques involution. With 61 or 62 exceptions, we give an explicit geometric construction of an Enriques involution on these singular K3 surfaces. This construction combines Shioda-Inose structures (3B) and the base change approach from [Hulek and Schütt 2011, §3].

In Section 6 we discuss the problem of Galois action on Néron-Severi groups. In this context, a different picture arises for Enriques surfaces than for K3 surfaces. The paper concludes with a formulation of several interesting classification problems for Enriques surfaces and K3 surfaces.

\section{Automorphisms of K3 surfaces}

2A. Basics about K3 surfaces and Enriques surfaces. This paper is concerned with complex algebraic K3 surfaces and Enriques surfaces. Here we briefly review their basic properties. For details the reader is referred to [Barth et al. 2004, Chapter VIII]; information and examples relevant for this paper can also be found in [Hulek and Schütt 2011].

A $K 3$ surface $X$ is a smooth projective surface with trivial canonical bundle $\omega_{X} \cong \mathscr{O}_{X}$ that is simply connected. The classical example consists in a smooth quartic in $\mathbb{P}^{3}$; here we will mostly work with elliptic K3 surfaces and Kummer surfaces.

In terms of the Enriques-Kodaira classification of algebraic surfaces, a complex Enriques surface $Y$ is a smooth projective surface with vanishing irregularity $q(Y)=h^{1}\left(Y, O_{Y}\right)=0$ and $\omega_{Y}^{\otimes 2}=\mathscr{O}_{Y}$, but $\omega_{Y} \neq \mathscr{O}_{Y}$. Equivalently $Y$ is the quotient 
of a K3 surface $X$ by a fixed point free involution $\tau$. Conversely the K3 surface $X$ can be recovered as the universal covering of $Y$.

The Néron-Severi group $\mathrm{NS}(S)$ of an algebraic surface $S$ is the group of divisors up to algebraic equivalence. Here we identify divisors moving in families such as fibres of a fibration. The Néron-Severi group is finitely generated abelian; its rank is called the Picard number and denoted by $\rho(S)$. In essence, $\mathrm{NS}(S)$ encodes the discrete structure of the Picard group of $S$. The intersection pairing endows NS $(S)$ with a quadratic form that also induces the notion of numerical equivalence.

On a K3 surface algebraic and numerical equivalence coincide, and $\operatorname{NS}(S)$ is torsion-free. Equipped with the intersection form, it becomes an even lattice of signature $(1, \rho(S)-1)$, the Néron-Severi lattice. On an Enriques surface, however, algebraic and numerical equivalence do not coincide, as in $\mathrm{NS}(Y)$ there is twotorsion represented by the canonical divisor $K_{Y}$. The quotient gives the torsion-free group of divisors up to numerical equivalence:

$$
\operatorname{Num}(Y)=\operatorname{NS}(Y) /\left\{0, K_{Y}\right\}
$$

The intersection pairing endows $\operatorname{Num}(Y)$ with a lattice structure. Contrary to the $\mathrm{K} 3$ case, this lattice has always the same rank and abstract shape:

$$
\operatorname{Num}(Y)=U+E_{8}(-1), \quad \operatorname{rank}(\operatorname{Num}(Y))=10
$$

where $U$ denotes the hyperbolic plane $\mathbb{Z}^{2}$ with intersection pairing $\left(\begin{array}{ll}0 & 1 \\ 1 & 0\end{array}\right)$ and $E_{8}$ is the unique even unimodular positive-definite lattice of rank 8 . The -1 indicates that the sign of the intersection form is reversed so that $\operatorname{Num}(Y)$ has signature $(1,9)$ as predicted by the Hodge index theorem.

The Torelli theorem [Piatetski-Shapiro and Shafarevich 1971] reduces many investigations of complex $\mathrm{K} 3$ surfaces $X$ to a study of $H^{2}(X)$ with its different structures as lattice or Hodge structure. By the cycle class map, $H^{2}(X)$ contains an algebraic part coming from $\mathrm{NS}(X)$. The orthogonal complement of $\mathrm{NS}(X)$ in $H^{2}(X, \mathbb{Z})$ is called the transcendental lattice:

$$
T(X)=\mathrm{NS}(X)^{\perp} \subset H^{2}(X, \mathbb{Z}) .
$$

As another characterisation, $T(X)$ is the smallest primitive sublattice of $H^{2}(X, \mathbb{Z})$ that contains the (up to scalar unique) 2-form $\eta_{X}$ after complexifying.

2B. Surfaces over number fields. We will consider complex surfaces $S$ that admit a model over some number field. This arithmetic setting brings up the natural question whether geometric objects such as $\mathrm{NS}(S)$ or the automorphism group $\operatorname{Aut}(S)$ are defined over the same field. The problem is as follows: 
Let $X$ be a complex K3 surface defined over a number field $L$. The action of its absolute Galois group $G_{L}=\operatorname{Gal}(\bar{L} / L)$ on NS $(X)$ factors through a finite extension $M / L$. We say that $\mathrm{NS}(X)$ is defined over $L$ if $M=L$, i.e., if $G_{L}$ acts trivially on $\operatorname{NS}(X)$. Throughout this paper, we will verify this property by exhibiting a set of generators of $\mathrm{NS}(X)$ each of which is defined over $L$. In fact, for elliptic surfaces with section (which we will mostly be concerned with), both conditions are equivalent.

The same terminology is employed for an Enriques surface $Y$ by saying that $\operatorname{NS}(Y)$ or $\operatorname{Num}(Y)$ is defined over a number field $L$ if $G_{L}$ acts trivially.

Let $\psi$ be an automorphism of a complex K3 surface $X$. Since we assumed $X$ to be algebraic, the induced automorphism $\psi^{*}$ acts as multiplication by a root of unity $\zeta$ on the holomorphic 2 -form $\eta_{X}$. We assume that $X$ is defined over some number field. The next proposition gives a criterion for the field of definition of $\psi$. This criterion will be crucial for the proof of Theorem 1.1.

Proposition 2.1. Let $X$ be a $K 3$ surface over some number field L. Let $\psi \in \operatorname{Aut}(X)$ and $\zeta \in \overline{\mathbb{Q}}$ such that $\psi^{*} \eta_{X}=\zeta \eta_{X}$. Assume that $\mathrm{NS}(X)$ is defined over $L$ and $\zeta \in L$. Then $\psi$ is defined over $L$.

Proof. We first need to show that $\psi$ is defined over some number field. Essentially this holds true because the automorphism group of any algebraic K3 surface is discrete by [Sterk 1985, Theorem 0.1]. The general idea is well-known: if the field of definition of $\psi$ were to require a transcendental extension of $L$, then the transcendental generators of this extension could be turned into parameters, so that $\psi$ would come in a nondiscrete family of automorphisms.

Now suppose that $\psi$ is defined over some finite extension $M / L$. We want to apply the Torelli theorem [Piatetski-Shapiro and Shafarevich 1971] to $\psi$ and its conjugates to deduce that $M=L$. For this purpose, we assume without loss of generality that $M / L$ is Galois. Let $\sigma \in \operatorname{Gal}(M / L)$. Then $\psi^{\sigma} \in \operatorname{Aut}(X)$, and we claim that $\psi=\psi^{\sigma}$. Explicitly we can write

$$
\psi^{\sigma}=\sigma \circ \psi \circ \sigma^{-1} \text {. }
$$

By the Torelli theorem, it suffices to verify the claim for the induced action on $\mathrm{NS}(X)$ and $T(X)$. For $\mathrm{NS}(X)$ this follows directly from the fact that $\sigma$ and $\sigma^{-1}$ act trivially by assumption. For $T(X)$, it suffices to check the action on the holomorphic 2-form. One has

$$
\left(\psi^{\sigma}\right)^{*}\left(\eta_{X}\right)=\left(\sigma^{-1}\right)^{*} \circ \psi^{*}\left(\eta_{X}\right)=\left(\sigma^{-1}\right)^{*}\left(\zeta \eta_{X}\right)=\zeta^{\sigma} \eta_{X}=\psi^{*}\left(\eta_{X}\right)
$$

since $\zeta \in L$. Hence $\psi^{*}=\left(\psi^{\sigma}\right)^{*}$ on $H^{2}(X, \mathbb{Z})$, and the claim $\psi=\psi^{\sigma}$ follows from the Torelli theorem [Piatetski-Shapiro and Shafarevich 1971]. In consequence, $\psi$ is defined over $L$. 
Remark 2.2. The conditions of Proposition 2.1 are sufficient, but not necessary. For instance, we exhibited a K3 surface with an Enriques involution over $\mathbb{Q}$, but with NS $(X)$ defined over $\mathbb{Q}(\sqrt{-3})$ in [Hulek and Schütt 2011, §5.3] (see also 6C).

2C. Enriques involutions. Proposition 2.1 has an immediate impact on involutions, and in particular on Enriques involutions. Namely for an involution $\psi$, the eigenvalue of $\eta_{X}$ can only be $\zeta= \pm 1$, so Proposition 2.1 only requires the NéronSeveri group of the covering K3 surface to be defined over $L$ :

Corollary 2.3. Let $X$ be a K3 surface over some number field L. If $\mathrm{NS}(X)$ is defined over $L$, then so is every involution on X. In particular, this holds for Enriques involutions.

Theorem 1.1 requires some concepts that we will discuss in detail in the next section. It concerns K3 surfaces with Picard number 20, the so-called singular $K 3$ surfaces (see 3A). By definition, the discriminant of a singular K3 surface $X$ is the determinant of the intersection form on $\mathrm{NS}(X)$. For a singular $\mathrm{K} 3$ surface, the discriminant $d$ gives rise to a very particular number field, the ring class field $H(d)$ as we discuss in 3D. In order to deduce Theorem 1.1, it suffices to combine Corollary 2.3 with the following result for any singular K3 surface (admitting an Enriques involution):

Theorem 2.4. Let $X$ be a singular K3 surface of discriminant $d$. Consider the ring class field $H(d)$. Then $X$ admits a model over $H(d)$ with $\mathrm{NS}(X)$ defined over $H(d)$.

The statement about a model over the ring class field $H(d)$ has been known before (cf. [Schütt 2007, Proposition 4.1]), but the extension for the Néron-Severi group seems to have gone unnoted until now. A proof will be given in the next two sections after reviewing the previous relevant results on singular K3 surfaces. We conclude this section with a direct corollary:

Corollary 2.5. Let $Y$ be an Enriques surface whose universal cover $X$ is a singular $K 3$ surface. Let $d<0$ denote the discriminant of $X$. Then $Y$ admits a model over the ring class field $H(d)$ with $\operatorname{Num}(Y)$ defined over $H(d)$.

The corresponding statement for $\mathrm{NS}(Y)$ does not hold true in general, as we will discuss within the framework of Galois actions on divisors in 6D. See Example 6.10 and Corollary 6.14.

\section{Arithmetic of singular K3 surfaces}

This section will review those parts of the theory of singular K3 surface that are relevant to our issues. The section culminates in Lemma 3.7, the main step towards the proof of Theorem 2.4. It is based on Shioda-Inose structures and Inose's fibration. All the required techniques will be explained along the way. 
3A. Singular K3 surfaces. A complex K3 surface $X$ is called singular if its Picard number $\rho(X)=\operatorname{rank} \mathrm{NS}(X)$ equals the maximum number allowed by Lefschetz's theorem:

$$
\rho(X)=h^{1,1}(X)=20 .
$$

Singular K3 surfaces involve no moduli, so the terminology "singular" should be understood in the sense of exceptional (just like for singular j-invariants of elliptic curves with complex multiplications, a similarity that will become clear very soon). We will discuss fields of definition of singular K3 surfaces in 3D. Recently singular K3 surfaces over $\mathbb{Q}$ have gained some prominence due to modularity; namely, in analogy with the Eichler-Shimura correspondence between modular forms of weight 2 and elliptic curves over $\mathbb{Q}$, for any suitable modular form of weight 3 there is a singular K3 surface over $\mathbb{Q}$ associated (cf. [Elkies and Schütt 2008b]).

By the Torelli theorem [Piatetski-Shapiro and Shafarevich 1971; Shioda and Inose 1977], singular K3 surfaces are classified up to isomorphism by their transcendental lattices. For a singular K3 surface, the transcendental lattice is even and positive definite of rank two and endowed with an orientation. Up to conjugation in $\mathrm{SL}_{2}(\mathbb{Z})$, we identify it with the quadratic intersection form

$$
Q(X)=\left(\begin{array}{cc}
2 a & b \\
b & 2 c
\end{array}\right)
$$

with integer entries $a, c \in \mathbb{N}, b \in \mathbb{Z}$ and discriminant $d=b^{2}-4 a c<0$. This number equals the determinant of the intersection form on $\mathrm{NS}(X)$; we refer to it as the discriminant of $X$. By the Torelli theorem [Piatetski-Shapiro and Shafarevich 1971; Shioda and Inose 1977] two singular K3 surfaces are isomorphic if and only if the transcendental lattices admit an isometry preserving the orientation (or equivalently the quadratic forms are conjugate in $\mathrm{SL}_{2}(\mathbb{Z})$ ).

The classical example for a singular K3 surface is the Fermat quartic in $\mathbb{P}^{3}$. Here we give an alternative example in terms of an elliptic fibration that will reappear later in another context $(5 \mathrm{G})$. Our treatment draws on the theory of elliptic surfaces; all relevant concepts can be found in [Schütt and Shioda 2010] for instance.

Example 3.1. Consider the universal elliptic curve for $\Gamma_{1}(6)$ :

$$
\mathscr{E}: y^{2}+(t-2) x y-t(t-1) y=x^{3}-t x^{2} .
$$

Here a point of order six is given by $(0,0)$. $\mathscr{E}$ gives rise to a rational elliptic surface $S$ over $\mathbb{P}^{1}$. By Tate's algorithm [1975], $S$ has the following singular fibres in Kodaira's notation:

\begin{tabular}{ccccc}
\hline fibre & $I_{6}$ & $I_{3}$ & $I_{2}$ & $I_{1}$ \\
\hline$t$ & $\infty$ & 0 & 1 & -8 \\
\hline
\end{tabular}


Any quadratic base change $f$ of $\mathbb{P}^{1}$ gives rise to a K3 surface $X$. We generally have $\rho(X) \geq 18$ by the Shioda-Tate formula [Shioda 1990, Corollary 5.3], but one can increase the Picard number conveniently by inferring ramification points at singular fibres. For instance, setting $t=-8 s^{2} /\left(s^{2}-1\right)$ yields an elliptic K3 surface $X$ with three singular fibres of type $I_{2}$ and $I_{6}$ each, and thus $\rho(X)=20$ over $\mathbb{C}$ again by the Shioda-Tate formula and the Lefschetz inequality $\rho(X) \leq h^{1,1}(X)$. On $X$, there are two additional two-torsion sections with $x$-coordinate

$$
-4 s^{2}(3 s \pm 1)(s \mp 1) /\left(s^{2}-1\right)^{2} .
$$

General theory shows that the singular fibres do not allow any further torsion in the Mordell-Weil group. Over $\mathbb{C}$ one obtains $\operatorname{MW}(X)=\mathbb{Z} / 2 \mathbb{Z} \times \mathbb{Z} / 6 \mathbb{Z}$. It follows that $X$ is the universal elliptic curve for the group $\Gamma_{1}(6) \cap \Gamma(2)$. By [Schütt and Shioda $2010,11.10(22)], N S(X)$ has discriminant -12 . With the discriminant form à la Nikulin [1980, Proposition 1.6.1 and Corollary 1.9.4], one can then compute the transcendental lattice with intersection form $Q(X)=\operatorname{diag}(2,6)$ (in agreement with the tables in [Shimada and Zhang 2001]).

3B. Shioda-Inose structure. In order to prove the surjectivity of the period map, mathematicians first considered Kummer surfaces. However, singular abelian surfaces (with $\rho(A)=4$ ) cannot possibly yield all singular K3 surfaces as Kummer surfaces because the transcendental lattice of a Kummer surface is always twodivisible as an even lattice. In detail, the intersection form is obtained from $T(A)$ by multiplication by 2 :

$$
T(\operatorname{Km}(A))=T(A)(2) .
$$

This problem of nonprimitivity was overcome by Shioda and Inose [1977]. Generally they considered two elliptic curves $E, E^{\prime}$. Their product is an abelian surface $A=E \times E^{\prime}$ and yields the Kummer surface $X^{\prime}=\operatorname{Km}\left(E \times E^{\prime}\right)$. Over $\mathbb{C}$, the Picard numbers depend on whether $E$ and $E^{\prime}$ are isogenous $\left(E \sim E^{\prime}\right)$ or have complex multiplication (CM):

$$
\begin{aligned}
\rho(A) & = \begin{cases}2 & \text { if } E \nsim E^{\prime}, \\
3 & \text { if } E \sim E^{\prime} \text { without CM, } \\
4 & \text { if } E \sim E^{\prime} \text { with } \mathrm{CM},\end{cases} \\
\rho\left(X^{\prime}\right) & =\rho(A)+16 .
\end{aligned}
$$

The Kummer surface $X^{\prime}$ admits several jacobian elliptic fibrations. For instance, the projections onto the factors $E$ and $E^{\prime}$ induce two isotrivial elliptic fibrations on the Kummer surface $X^{\prime}$ that we will analyse in Section 4. In [Shioda and Inose $1977, \S 2]$, a jacobian elliptic fibration with a fibre of type $I I^{*}$ was found on $X^{\prime}$. It 
has exactly two further reducible fibres of the following types:

$$
\begin{array}{ll}
2 I_{0}^{*} & E \varsubsetneqq E^{\prime}, \\
I_{0}^{*}, I_{1}^{*} & E \cong E^{\prime}, j(E) \neq 0,12^{3}, \\
2 I_{1}^{*} & j(E)=j\left(E^{\prime}\right)=12^{3}, \\
I_{0}^{*}, I V^{*} & j(E)=j\left(E^{\prime}\right)=0 .
\end{array}
$$

Starting from this elliptic fibration, we proceed with the quadratic base change

$$
f: \mathbb{P}^{1} \rightarrow \mathbb{P}^{1}
$$

that ramifies exactly at the above two reducible singular fibres. Since both ramified fibres are nonreduced, the base change applied to $X^{\prime}$ results in another elliptic K3 surface $X$. By construction, the elliptic K3 surface $X$ has two fibres of type $I I^{*}$ and possibly some reducible fibres of type $I_{2}$ or $I V$ depending on the above cases. The Kummer surface $X^{\prime}$ can be recovered from $X$ as (the desingularisation of) the quotient by the involution of the double cover $X \rightarrow X^{\prime}$. (In [Hulek and Schütt 2011] we abused terminology by referring to this involution as deck transformation, but here we will call it base change involution.) The base change involution is a Nikulin involution that composes the involution on the base curve $\mathbb{P}^{1}$ with the hyperelliptic involution on the fibres:

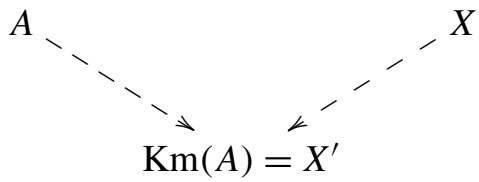

The gist of this construction is that the $\mathrm{K} 3$ surface $X$ recovers the transcendental lattice of the abelian surface $A$ :

$$
T(X)=T\left(X^{\prime}\right)(1 / 2)=T(A) .
$$

Morrison coined the terminology Shioda-Inose structure for such a setting: abelian surface and K3 surface with the same transcendental lattice such that Kummer quotient and Nikulin involution yield the same Kummer surface. He developed lattice theoretic criteria to decide which K3 surfaces of Picard number $\rho \geq 17$ admit a Shioda-Inose structure [Morrison 1984, §6].

3C. Surjectivity of the period map. The surjectivity of the period map requires to exhibit singular K3 surfaces for any quadratic form $Q$ as in (1). By the above considerations, this can be achieved by exhibiting a singular abelian surface $A$ with $Q(A)=Q$ because then the Shioda-Inose structure provides a suitable singular K3 surface $X$ with $Q(X)=Q$. 
Chronologically, the corresponding surjectivity statement for singular abelian surfaces was already established before Shioda-Inose's work by Shioda and Mitani [1974]. Namely, it was shown that any singular abelian surface has product type. Given the quadratic form $Q(A)$ with coefficients as in (1), the abelian surface $A$ admits the representation $A=E \times E^{\prime}$ with the following elliptic curves given as complex tori $E_{\tau}=\mathbb{C} /(\mathbb{Z}+\tau \mathbb{Z})$ :

$$
E=E_{\tau}, \quad \tau=\frac{-b+\sqrt{d}}{2 a}, \quad E^{\prime}=E_{\tau^{\prime}}, \quad \tau^{\prime}=\frac{b+\sqrt{d}}{2} .
$$

Note that this representation need not be unique, and in fact there can be arbitrarily many distinct representations for the same singular abelian surface (and thus also for singular K3 surfaces).

Example 3.2. The K 3 surface $X$ from Example 3.1 is not a Kummer surface, since $T(X)$ is not two-divisible as an even lattice. Through the Shioda-Inose structure, $X$ arises from the self-product of the elliptic curve $E_{\sqrt{-3}}$ with j-invariant $2^{4} 3^{3} 5^{3}$.

3D. Fields of definition. We have seen that every singular abelian surface $A$ is the product of two elliptic curves with CM in the same field. CM elliptic curves are well-understood thanks to the connection to class field theory (cf. [Shimura 1971, $\S 5])$. Indeed both curves in (4) are defined over the ring class field $H(d)$. This field is an abelian Galois extension of the imaginary quadratic field $K=\mathbb{Q}(\sqrt{d})$ with prescribed ramification and Galois group isomorphic to the class group $\mathrm{Cl}(d)$ (see [Cox 1989, §9]). We recall one way to describe $C l(d)$ : it consists of $\mathrm{SL}_{2}(\mathbb{Z})$ conjugacy classes of primitive $2 \times 2$ matrices $Q$ as in (1) of discriminant $d<0$ together with Gauss composition (cf. [Cox 1989, §3] for instance). By [Shimura 1971, Theorem 5.7], $H(d)$ is generated over $K$ by adjoining the j-invariant of $E^{\prime}$, or in fact of any elliptic curve with CM by the given order in $K$ of discriminant $d$. Here $C l(d)$ acts naturally as a permutation on all these CM elliptic curves abstractly on the complex tori, but also in a compatible way through the Galois action on $H(d)$ permuting j-invariants.

Shioda-Inose used these CM properties to deduce that any singular K3 surface is defined over some number field. Namely, the Kummer quotient $X^{\prime}$ respects the base field (a property that we will exploit in Section 4). Hence the only step in the Shioda-Inose structure that may require increasing the base field concerns the elliptic fibration with a fibre of type $I I^{*}$.

Subsequently Inose [1978] exhibited an explicit model for $X$ over a specific extension of $H(d)$. This model is expressed purely in terms of the j-invariants $j, j^{\prime}$ of the elliptic curves $E, E^{\prime}$ from (4):

$$
X: y^{2}=x^{3}-3 A t^{4} x+t^{5}\left(t^{2}-2 B t+1\right),
$$


where $A^{3}=j j^{\prime} / 12^{6}$ and $B^{2}=\left(1-j / 12^{3}\right)\left(1-j^{\prime} / 12^{3}\right)$. Thus we know that any singular K3 surface $X$ of discriminant $d$ admits a model over a degree six extension of $H(d)$. In [Schütt 2007, Proposition 4.1] it was then noted that the above fibration can be twisted in such a way that it is defined over $H(d)$ (cf. (14) in case $A B \neq 0$ ):

Theorem 3.3. Let $X$ be a singular $K 3$ surface of discriminant $d$. Then $X$ has a model over the ring class field $H(d)$.

In practice, the given field of definition can be far from optimal, that is, $X$ may admit a model over a much smaller number field. In fact, the modularity converse in [Elkies and Schütt 2008b] required to exhibit models of singular K3 surfaces over $\mathbb{Q}$ where the ring class field had degree as large as 32 over $\mathbb{Q}$. We can already detect a similar behaviour on the level of the elliptic curves $E, E^{\prime}$ in (4): because of the Galois action of the class group $C l(d)$, the elliptic curve $E^{\prime}$ can at best be defined over a quadratic subfield of $H(d)$. The factor $E$, however, may be defined over $\mathbb{Q}$ even for large $d$ by inspection of the denominators in (4).

3E. Néron-Severi group. In the remainder of this section, we derive an important intermediate result for the proof of Theorem 2.4. The remaining steps will be done in 4F. We have recalled in Theorem 3.3 that any singular K3 surface $X$ admits a model over the ring class field $H(d)$. Here $d$ denotes the discriminant of $T(X)$ as usual. It remains to show that there always is a model of $X$ with $\operatorname{NS}(X)$ defined over $H(d)$ as well.

The basic idea for the proof is to work with a model of Inose's pencil (5) over $H(d)$ as in the proof of [Schütt 2007, Proposition 4.1]:

$$
X: \quad y^{2}=x^{3}+a t^{4} x+t^{5}\left(b_{2} t^{2}+b_{1} t+b_{0}\right), \quad a, b_{i} \in H(d) .
$$

Note that fibres of type $I I^{*}$ do not admit any inner Galois action (i.e. on fibre components). Hence these two singular fibres of $X$ together with the zero section generate a sublattice $U+2 E_{8}(-1) \subset \mathrm{NS}(X)$ that is fully defined over the base field $H(d)$. It remains to study the Galois action on the remaining generators of $\mathrm{NS}(X)$ (there are two generators remaining, since $\rho(X)=20$ ). Looking at the other reducible singular fibres, we distinguish four cases as in $3 \mathrm{~B}$ :

\begin{tabular}{ccl}
\hline Reducible fibres other than $I I^{*}$ & $\operatorname{rank}(\mathrm{MW})$ & case \\
\hline- & 2 & $E \nsubseteq E^{\prime}$ \\
$I_{2}$ & 1 & $E \cong E^{\prime}, j(E) \neq 0,12^{3}$ \\
$2 I_{2}$ & 0 & $E \cong E^{\prime}, j(E)=12^{3}$ \\
$I V$ & 0 & $E \cong E^{\prime}, j(E)=0$ \\
\hline
\end{tabular}

Table 1. Singular fibres and MW-rank of Inose's pencil. 
Lemma 3.4. If the singular $K 3$ surface $X$ admits an Inose pencil (5) of MW-rank at most one, then $X$ has a model with $\mathrm{NS}(X)$ defined over $H(d)$.

Proof. For the last two surfaces in Table 1 (MW-rank zero), there are explicit models with NS $(X)$ defined over $\mathbb{Q}$ (cf. [Schütt 2010, §10]). For the case of MWrank one with an $I_{2}$ fibre, it is also easy to see that $\mathrm{NS}(X)$ can be defined over $L=H(d)$. The fibre does not admit any Galois action, since the identity component is fixed by Galois. By the formula of Shioda-Tate, the Mordell-Weil group has rank one. The Mordell-Weil generator $P$ can only be either fixed or mapped to its inverse by Galois. But if the latter is the case, then the section $P$ is defined over some quadratic extension of $L$. More precisely, it is given in $x, y$-coordinates as $P=(U, \sqrt{\gamma} V)$ for some $\gamma \in L, U, V \in L(t)$. Consider the quadratic twist of $X$ with respect to this quadratic extension of $L$ :

$$
\gamma y^{2}=x^{3}+a t^{4} x+t^{5}\left(b_{2} t^{2}+b_{1} t+b_{0}\right) .
$$

This is an alternative model of the fixed elliptic fibration (6) on $X$ over $L$ such that both models become isomorphic over $L(\sqrt{\gamma})$. This quadratic twist transforms the section to $(U, V)$ (defined over $L$ ) without introducing any Galois action on the singular fibres (since they only have types $I_{1}, I_{2}, I I, I I^{*}$ ). Thus the Néron-Severi group of the new model of $X$ is defined over $L=H(d)$.

Remark 3.5. If $T(X)$ is primitive and lies in the principal genus, then it is possible to replace the CM-curves $E, E^{\prime}$ by opposite Galois conjugates that are isomorphic: $E^{\sigma} \cong\left(E^{\prime}\right)^{\sigma^{-1}}$. By [Schütt 2007, §6] (which combines [Shimura 1971] and [Shioda and Mitani 1974]), one has $T\left(E^{\sigma} \times\left(E^{\prime}\right)^{\sigma^{-1}}\right)=T\left(E \times E^{\prime}\right)$. According to Table 1, the induced Inose pencil on $X$ has MW-rank one. By Lemma 3.4 this produces a model of $X$ with $\mathrm{NS}(X)$ defined over $H(d)$.

3F. Mordell-Weil lattices. A similar argument goes through for almost all instances of the case where $E ¥ E^{\prime}$. Here we can argue with the Mordell-Weil lattice $\operatorname{MWL}(X)$ of the fibration. In general, the Mordell-Weil lattice of an elliptic surface $S \rightarrow C$ with section was defined in [Shioda 1990] as follows. In NS $(S)$ consider the trivial lattice $\operatorname{Triv}(S)$ generated by the zero section and fibre components. By [Shioda 1990, Theorem 1.3] there is an isomorphism

$$
\operatorname{MW}(S) \cong \mathrm{NS}(S) / \operatorname{Triv}(S)
$$

The torsion in $\mathrm{MW}(S)$ is contained in (and determined by) the primitive closure $\operatorname{Triv}(S)^{\prime}$ of $\operatorname{Triv}(S)$ inside $\mathrm{NS}(S)$. The quotient $\mathrm{MW}(S) / \mathrm{MW}(S)_{\text {tor }}$ is endowed with a lattice structure by means of the orthogonal projection $\varphi$ in $\operatorname{NS}(S)_{\mathbb{Q}}$ with respect to $\operatorname{Triv}(S)$. Here tensoring with $\mathbb{Q}$ is required unless $\operatorname{Triv}(S)^{\prime}$ is unimodular. By construction $\varphi(\mathrm{MW}(S))(-1)$ is a positive definite, though not necessarily 
integral lattice that one refers to as Mordell-Weil lattice $\operatorname{MWL}(S)$. The MordellWeil lattice satisfies functorial properties for base change and Galois actions. For details the reader is referred to [Shioda 1990] or the survey paper [Schütt and Shioda 2010].

In the present situation the only reducible fibres have type $I I^{*}$. The nonidentity fibre components generate the root lattice $E_{8}(-1)$, so $\operatorname{Triv}(X)=U+2 E_{8}(-1)$. Hence $\operatorname{MWL}(X)$ is a positive definite even integral lattice of rank two that fits into the decomposition

$$
\mathrm{NS}(X)=U+2 E_{8}(-1)+\operatorname{MWL}(X)(-1) .
$$

Since $\operatorname{Triv}(X)$ is unimodular, the discriminant forms of $\operatorname{NS}(X)$ and $\operatorname{MWL}(X)$ agree up to sign. By [Nikulin 1980, Corollary 1.9.4], this implies that $T(X)$ and $\operatorname{MWL}(X)$ lie in the same genus (or in the same isogeny class).

3G. Binary even quadratic forms. To understand the possible Galois actions on $\operatorname{MWL}(X)$, we shall need a simple observation about the automorphisms of such lattices. It will be phrased in terms of the corresponding quadratic form $Q$ as in (1). Multiplication by \pm 1 gives the trivial automorphisms of $Q$; any other automorphism will be called nontrivial. The problem whether $Q$ admits nontrivial automorphisms depends on its order in the class group of even positive definite binary quadratic forms with given discriminant and degree of primitivity:

Lemma 3.6. The positive-definite quadratic form $Q$ admits a nontrivial automorphism if and only if it is two-torsion in its class group.

The proof is elementary, so we will omit it here although we did not find a concise reference. For later use, we shall give the possible automorphism groups. Recall that any quadratic form $Q$ as in (1) can be transformed by conjugation in $S L_{2}(\mathbb{Z})$ to a reduced form where the coefficients satisfy $-a<b \leq a \leq c$ (and $b \geq 0$ if $a=c$ ). The inverse of a quadratic form is obtained by replacing $b$ by $-b$. A reduced quadratic form is two-torsion if and only if

$$
b=0 \text { or } a=b \text { or } a=c .
$$

We obtain the following nontrivial automorphism groups where $D_{2 n}$ denotes the dihedral group of order $2 n$ :

3H. Intermediate step. We conclude this section with an intermediate result towards the proof of Theorem 2.4. In the next section, we will use the Shioda-Inose structure to complete the proof.

Lemma 3.7. In all cases of MW-rank two in Table 1, the model (5) admits a twist such that there is an $H(d)$-rational section. 


\begin{tabular}{|c|c|c|c|c|c|}
\hline$Q$ & $\begin{array}{c}\left(\begin{array}{cc}2 a & 0 \\
0 & 2 c\end{array}\right) \\
a<c\end{array}$ & $\begin{array}{c}\left(\begin{array}{cc}2 a & a \\
a & 2 c\end{array}\right) \\
a<c\end{array}$ & $\begin{array}{l}\left(\begin{array}{cc}2 a & b \\
b & 2 a\end{array}\right) \\
0<b<a\end{array}$ & $\left(\begin{array}{cc}2 a & 0 \\
0 & 2 a\end{array}\right)$ & $\left(\begin{array}{cc}2 a & a \\
a & 2 a\end{array}\right)$ \\
\hline $\operatorname{Aut}(Q)$ & $(\mathbb{Z} / 2 \mathbb{Z})^{2}$ & $(\mathbb{Z} / 2 \mathbb{Z})^{2}$ & $(\mathbb{Z} / 2 \mathbb{Z})^{2}$ & $D_{8}$ & $D_{12}$ \\
\hline
\end{tabular}

Table 2. Quadratic forms with nontrivial automorphisms groups.

Proof. If the automorphism group of MWL is only two-torsion, then the lemma follows after a quadratic twist for one of the MW generators. This leaves the cases of the last two quadratic forms in Table 2. Here the class number of $Q$ is one. Hence $T(X)$ has exactly the intersection form $Q$. In the Shioda-Inose structure, we can choose $E$ by (4) with j-invariant $j=12^{3}$ resp. $j=0$. The extra automorphism of $E$ induces an extra automorphism on $X$ that respects the elliptic fibration (5):

$$
(x, y, t) \mapsto(-x, i y,-t) \text { resp. } \quad(x, y, t) \mapsto(\varrho x, y, t)
$$

where $\varrho, i$ denote primitive third resp. fourth roots of unity. The respective automorphism makes $\operatorname{MWL}(X)$ into a module of rank one over $\mathbb{Z}[i]$ resp. $\mathbb{Z}[\varrho]$. This identification is compatible with the Galois action over $H(d)$, since the automorphisms are defined over $H(d)$. Hence it suffices to study the Galois action on the given modules of rank one. Their only automorphisms are the units in $\mathbb{Z}[i]$ resp. $\mathbb{Z}[\varrho]$, i.e. the group of fourth resp. sixth roots of unity. On the elliptic curves with CM by these rings, it is well-known that such a Galois action can be accounted for by biquadratic or sextic twisting (see [Silverman 1994, §II, Example 10.6 and Exercises 2.33, 2.34] or [Schütt 2008, §8]). Thanks to the special shape of the present Weierstrass form (5) with $A=0$ or $B=0$, this translates directly into twists of $X$. Thus there is a twist with $\operatorname{MWL}(X)$ defined over $H(d)$.

Remark 3.8. If MWL admits no nontrivial automorphisms, then Lemma 3.7 already settles Theorem 2.4 completely. By the proof of Lemma 3.7, this also holds for MWL with nonabelian automorphism group (the last two entries in Table 2). It is the two-torsion cases of Table 2 that require an extra argument.

In the next section, we will use the Shioda-Inose structures and study Kummer surfaces of product type in detail. In this case, although we may not have any automorphisms on the Kummer surface to relate the MW-generators, we can use the endomorphisms of the abelian surface instead. This approach will enable us to complete the proof of Theorem 2.4 in $4 \mathrm{~F}$.

\section{Singular Kummer surfaces of product type}

Let $E, E^{\prime}$ be isogenous complex elliptic curves with $\mathrm{CM}$. Then the abelian surface $A=E \times E^{\prime}$ is singular $(\rho(A)=4)$ ). Let $d$ denote its discriminant (that is the 
discriminant of $T(A))$. Then $E, E^{\prime}$ have models over the ring class field $H(d)$ (obtained from the CM-field by adjoining the $\mathrm{j}$-invariants).

Throughout this section, we only consider the case where $E \nsubseteq E^{\prime}$ (MW-rank two) and no j-invariant equals 0 or $12^{3}$ (no extra automorphisms). The same results hold in the other cases, but we would have to distinguish more subcases and also consider biquadratic/sextic twisting etc. Note that for the excluded cases we have already given a full proof of Theorem 2.4 in Lemma 3.4 (for $E \cong E^{\prime}$ ) and in the proof of Lemma 3.7 (for $j$ or $j^{\prime} \in\left\{0,12^{3}\right\}$; cf. Remark 3.8). Thus the cases considered explicitly in this section will suffice to complete the proof of Theorem 2.4.

4A. Consider the Kummer surface $X^{\prime}=\operatorname{Km}(A)$. Recall the isotrivial elliptic fibrations on $X^{\prime}$ that are induced by the projections onto $E$ and $E^{\prime}$ from $3 \mathrm{~B}$. These are naturally defined over $H(d)$ as follows. Fix Weierstrass models

$$
E: y^{2}=f(x), \quad E^{\prime}: y^{2}=g(x)
$$

with cubic polynomials $f, g \in H(d)[x]$. Then $X^{\prime}$ admits a birational model

$$
X^{\prime}: \quad f(t) y^{2}=g(x)
$$

with the structure of an elliptic curve over the function field $H(d)(t)$. We denote the corresponding elliptic fibration by the pair $\left(X^{\prime}, \pi\right)$. This fibration has singular fibres of type $I_{0}^{*}$ at $\infty$ and at the zeroes of $f(t)$. Over $\overline{\mathbb{Q}}$ we have $\operatorname{MW}\left(X^{\prime}, \pi\right)=$ $\mathbb{Z}^{2} \times(\mathbb{Z} / 2 \mathbb{Z})^{2}$ with torsion sections given by the roots of $g(x)$.

Proposition 4.1. The elliptic fibration $\left(X^{\prime}, \pi\right)$ admits a model over $H(d)$ such that MW is generated by two-torsion and sections defined over $H(d)$. In particular, MWL is generated by sections defined over $H(d)$.

Proof. By the Shioda-Tate formula, the Mordell-Weil lattice has rank two since $\rho\left(X^{\prime}\right)=20$. Due to the singular fibre types $\operatorname{MWL}\left(X^{\prime}, \pi\right)$ will not be integral, but it is positive-definite. Hence the results from $3 \mathrm{G}$ and $3 \mathrm{H}$ apply directly to prove the claim with the exception of the first three special cases from Table 2. Here we pursue an alternative uniform approach based on the fact that as in Lemma 3.7 we can find a quadratic twist with at least one MW-generator $P$ over $H(d)$.

The crucial ingredient is the following lattice isomorphism which Shioda established in [Shioda 2007, Proposition 3.1]:

$$
\operatorname{Hom}\left(E, E^{\prime}\right) \cong \operatorname{MWL}\left(X^{\prime}, \pi\right) .
$$

$\operatorname{Here} \operatorname{Hom}\left(E, E^{\prime}\right)$ is endowed with a norm given by the degree. The isomorphism takes a homomorphism $\phi: E \rightarrow E^{\prime}$ as input. Via its graph $\Gamma_{\phi}$ in $A$ and the image $\bar{\Gamma}_{\phi}$ in $X^{\prime}$, one associates to $\phi$ the element $\bar{R}_{\phi}$ in $\operatorname{MWL}\left(X^{\prime}, \pi\right)$ corresponding to $\bar{\Gamma}_{\varphi}$ under the orthogonal projection $\operatorname{NS}\left(X^{\prime}\right) \rightarrow \operatorname{MWL}\left(X^{\prime}, \pi\right)$ (see $3 \mathrm{~F}$ ). 
Shioda [2007] worked over an algebraically closed field, so that the isomorphism (9) is independent of the chosen model. However, for the specified models in (7), (8) the isomorphism (9) is clearly Galois-equivariant.

Following Lemma 3.7, we apply a quadratic twist on $X^{\prime}$ such that there is an $H(d)$-rational section $P$ (nontorsion). That is, for some $c \in H(d)$ we consider the $H(d)(\sqrt{c})$-isomorphic model

$$
X^{\prime}: c f(t) y^{2}=g(x) .
$$

In terms of the elliptic curves $E, E^{\prime}$, this is accounted for by twisting one elliptic curve by $\sqrt{c}$, say:

$$
E: y^{2}=f(x), \quad E^{\prime}: c y^{2}=g(x) .
$$

For these models, the isomorphism (9) is by construction again Galois-equivariant. Hence the section $P$ corresponds to a homomorphism $\phi: E \rightarrow E^{\prime}$ over $H(d)$. Now pick any endomorphism $\epsilon$ of $E^{\prime}$ that is not multiplication by an integer. By CMtheory, $\epsilon$ is defined over $H(d)$, and together $\phi, \epsilon \circ \phi$ generate the lattice $\operatorname{Hom}\left(E, E^{\prime}\right)$ up to finite index. In conclusion, (9) gives a section $R_{\epsilon \circ \phi}$ over $H(d)$ that is independent of $P$. By construction, these sections generate $\operatorname{MWL}\left(X^{\prime}, \pi\right)$ up to finite index. Proposition 4.1 thus follows.

4B. Néron-Severi group of Kummer surfaces. We collect a few consequences of Proposition 4.1. We start with a version of Theorem 6.3 for singular Kummer surfaces. Note that since $T\left(X^{\prime}\right)=T(A)(2)$, the Kummer surface $X^{\prime}$ has discriminant $4 d$.

Corollary 4.2. The singular Kummer surface $X^{\prime}$ has a model over $H(d)$ with $\mathrm{NS}\left(X^{\prime}\right)$ defined over $H(4 d)$.

Proof. Fix the model of the elliptic fibration $\left(X^{\prime}, \pi\right)$ from Proposition 4.1 with MW-rank two over $H(d)$. In order to generate $\mathrm{NS}\left(X^{\prime}\right)$, we have to add to these $H(d)$-rational sections the two-torsion sections and the components of the $I_{0}^{*}$ fibres. These rational curves are defined over the splitting field of the polynomials $f(t), g(x)$ over $H(d)$. That is, we adjoin to $H(d)$ the $x$-coordinates of the twotorsion points of $E$ and $E^{\prime}$. By the analogue of the Kronecker-Weber theorem for imaginary quadratic number fields [Silverman 1994, §II Theorem 5.6], these algebraic numbers generate exactly $H(4 d)$ over $H(d)$.

4C. Isogenous CM-elliptic curves. Before continuing with the proof of Theorem 2.4, we note another implication of Proposition 4.1. Here we are concerned with the field of definition of the isogeny between $E$ and $E^{\prime}$. By the classical theory, any two elliptic curves with $\mathrm{CM}$ in the same field $K$ have models over some minimal ring class field $H$; moreover they are isogenous over $\overline{\mathbb{Q}}$. Here we ask whether they 
admit $H$-isogenous models, i.e. models over $H$ with isogeny defined over $H$ as well. When the $C M$-curves are $\mathbb{Q}$-curves, this property comes for free, but this situation does not always persist (cf. Remark 4.4). The following result might be well-known to the experts, but we could not find a reference.

Corollary 4.3. Let $E, E^{\prime}$ be elliptic curves with $C M$ by orders in the same imaginary quadratic field $K$. Let $H=K\left(j(E), j\left(E^{\prime}\right)\right)$. Then $E$, $E^{\prime}$ have $H$-isogenous models.

Proof. We can start with any two Weierstrass forms over $H$ as in (7). The proof of Proposition 4.1 exhibits a quadratic twist of $E^{\prime}$ with a nontrivial homomorphism $\phi: E \rightarrow E^{\prime}$.

Remark 4.4. Corollary 4.3 only seemingly conflicts with a result of Gross [1980, $\S 11]$. Namely, Gross found that there are CM-elliptic curves which are not $\mathbb{Q}$ curves, i.e. $E$ is not $H$-isogenous to all its conjugates. Here we let $E^{\prime}=E^{\sigma}$ be a conjugate of $E$. If $E, E^{\sigma}$ are not $H$-isogenous (so that $E$ is not a $\mathbb{Q}$-curve), then Corollary 4.3 provides us with a quadratic twist of $E^{\sigma}$ which is $H$-isogenous to $E$. But then the quadratic twist of $E^{\sigma}$ and $E$ are not conjugate any more, so there is no contradiction to $E$ 's failure of being a $\mathbb{Q}$-curve.

4D. Auxiliary elliptic fibration. Recall the singular K3 surface $X$ with Inose's elliptic fibration (5). By [Shioda 2006] the quadratic base change $t=u^{2}$ recovers the Kummer surface $X^{\prime}$. Since $X$ also dominates $X^{\prime}$ by the Shioda-Inose structure, Shioda alluded to this picture as $X$ being sandwiched by the Kummer surface $X^{\prime}$. In the base change, the two fibres of type $I I^{*}$ are replaced by fibres of type $I V^{*}$. Let us explain how to find this base changed fibration on the previous model of $X^{\prime}$ :

$$
X^{\prime}: \quad c f(t) y^{2}=g(x) .
$$

Projection onto the affine coordinate $u=y$ endows $X^{\prime}$ with the structure of an elliptic fibration $\pi^{\prime}$ since the fibres are plane cubics in $x, t$. Write $\left(X^{\prime}, \pi^{\prime}\right)$ for $X^{\prime}$ with this fixed elliptic fibration. Visibly $\left(X^{\prime}, \pi^{\prime}\right)$ is the quadratic base change of the rational elliptic surface $S^{\prime}$ obtained by setting $u^{2}=v$. $S^{\prime}$ has singular fibres of type $I V$ at $v=0, \infty$; in $X^{\prime}$ they are replaced by fibres of type $I V^{*}$ as alluded to before. Here $S^{\prime}$ is given as a cubic pencil whose base points form sections. Recall that these sections are all defined over $H(4 d)$.

By base change $\operatorname{MWL}(S)(2)$ embeds into $\operatorname{MWL}\left(X^{\prime}, \pi^{\prime}\right)$. Consider the orthogonal complement

$$
L=\left[\operatorname{MWL}\left(S^{\prime}\right)(2)\right]^{\perp} \subset \operatorname{MWL}\left(X^{\prime}, \pi^{\prime}\right) .
$$

By construction, $L$ is exactly the invariant sublattice of $\operatorname{MWL}\left(X^{\prime}, \pi^{\prime}\right)$ for the involution corresponding to the base change $X^{\prime} \rightarrow X$, i.e. $L=\operatorname{MWL}(X)(2)$. 
Over $\overline{\mathbb{Q}}$ (or in fact algebraically closed fields of characteristic $\neq 2,3$ ), Shioda used a similar argument as for the isomorphism (9) to derive an isomorphism

$$
L \cong \operatorname{Hom}\left(E, E^{\prime}\right)(4), \text { so that } \operatorname{MWL}(X) \cong \operatorname{Hom}\left(E, E^{\prime}\right)(2) .
$$

Compared to the previous argument that gave (9), there is one subtlety here: For $\phi \in \operatorname{Hom}\left(E, E^{\prime}\right)$, the orthogonal projection onto $L_{\mathbb{Q}}$ maps the divisor $\bar{\Gamma}_{\phi}$ to $\frac{1}{2} L$. This holds true since the quotient $\operatorname{MWL}\left(X^{\prime}, \pi^{\prime}\right) /\left(L+L^{\perp}\right)$ need not be trivial (hence we tensor $L$ with $\mathbb{Q}$ a priori), but due to the quadratic base change the quotient is always isomorphic to a finite number of copies of $\mathbb{Z} / 2 \mathbb{Z}$. Now instead of $\bar{\Gamma}_{\phi}$, one takes the image of the divisor $2 \bar{\Gamma}_{\phi}$ in $L$. Computing intersection numbers using the theory of Mordell-Weil lattices, Shioda verifies the isomorphism (11). In our setting, the main problem is to find models which make the isomorphisms (11) Galois-equivariant over a suitable field.

4E. Galois equivariance. We know that $E, E^{\prime}$ admit $H(d)$-isogenous models, so that $\operatorname{Hom}\left(E, E^{\prime}\right)$ is generated by isogenies over $H(d)$. The elliptic fibration $\pi^{\prime}$ on $X^{\prime}$ is defined over $H(d)$ as well, but in order to endow it with a section (a base point of the cubic pencil), we may have to increase the base field to $H(4 d)$. This makes the isomorphisms in (11) for the specified models Galois-equivariant over $H(4 d)$. For $X$, however, we need a model with MWL over $H(d)$, so we have to throw in some more information. We distinguish two cases according to the degree $h$ of the Galois extension $H(4 d) / H(d)$. Note that with the Legendre symbol $(\cdot / 2)$ at 2 , one obtains from the class number formula

$$
h=\operatorname{deg}(H(4 d) / H(d))= \begin{cases}1 & \text { if }(d / 2)=1 \text { or } d=-3,-4 \\ 2 & \text { if } 2 \mid d, d \neq-4 \\ 3 & \text { if }(d / 2)=-1, d \neq-3 .\end{cases}
$$

4E.1. First case: $h=1,2$. This case is very simple. By assumption, both polynomials $f, g$ have a root over $H(d)$. A base point of the cubic pencil gives an $H(d)$-rational section of the elliptic fibration $\left(X^{\prime}, \pi^{\prime}\right)$. Due to the singular fibre types and the involution $u \mapsto-u$, we obtain a Weierstrass form

$$
X^{\prime}: y^{\prime 2}=x^{\prime 3}-3 a u^{4} x^{\prime}+u^{4}\left(b_{2} u^{4}-2 b_{1} u^{2}+b_{0}\right) .
$$

As quotient by the base change involution $u \mapsto-u$ of $X^{\prime} \rightarrow S^{\prime}$ composed with the hyperelliptic involution $y^{\prime} \mapsto-y^{\prime}$, we obtain a model of $X$ over $H(d)$. Compared to (5), this Weierstrass form is not yet normalised with respect to $b_{0}, b_{2}$.

By construction, the isomorphisms (11) are $H(d)$-Galois equivariant for these specific models of $E, E^{\prime}, X^{\prime}, X$. That is, we have exhibited a model of $X$ over $H(d)$ with fibration of type (5) and MW-rank two over $H(d)$. It follows that this model has $\mathrm{NS}(X)$ defined over $H(d)$. 
4E.2. Second case: $h=3$. In this case, we compare two $\overline{\mathbb{Q}}$-isomorphic models that we denote by $X_{1}, X_{2}$. From (12), we obtain a model over $H(4 d)$ as quotient by the Nikulin involution $\left(x^{\prime}, y^{\prime}, u\right) \mapsto\left(x^{\prime},-y^{\prime},-u\right)$ :

$$
X_{1}: y^{\prime 2}=x^{\prime 3}-3 a u^{4} x^{\prime}+u^{5}\left(b_{2} u^{2}-2 b_{1} u+b_{0}\right)
$$

with $\operatorname{MWL}\left(X_{1}\right)$ defined over $H(4 d)$ by the Galois-equivariant isomorphism (11). From (5), we derive a model over $H(d)$

$$
X_{2}: y^{2}=x^{3}-3 c^{2} B^{2} A^{3} t^{4} x+c^{3} B^{2} A^{3} t^{5}\left(B^{2} t^{2}-2 B^{2} t+1\right) .
$$

Here $B^{2}, A^{3} \in H(d)$ as given in 3B. By Lemma 3.7, we can choose $c \in H(d)$ in such a way that $X_{2}$ has an $H(d)$-rational section $P$ and an orthogonal section $Q$ defined over some quadratic extension $M$ of $H(d)$. We assume that $M \neq H(d)$ and derive a contradiction from the above two models. Essentially, this works because we compare a quadratic and a cubic extension of $H(d)$.

By assumption, we can choose $Q$ anti-invariant under conjugation in $M / H(d)$ (so that $P, Q$ generate $\mathrm{MW}\left(X_{2}\right)$ up to finite index). Hence there are rational functions $x_{Q}, y_{Q} \in H(d)(t)$ and some constant $c_{Q} \in H(d)$ such that

$$
Q=\left(x_{Q}, \sqrt{c_{Q}} y_{Q}\right) \text { and } M=H(d)\left(\sqrt{c_{Q}}\right) .
$$

We work out an isomorphism of the two elliptic fibrations $X_{1}, X_{2}$. This can only take the shape

$$
(x, y, t) \mapsto\left(x^{\prime}, y^{\prime}, u\right)=\left(\gamma \alpha^{2} x, \alpha^{3} \gamma^{3 / 2} y, \alpha t\right) .
$$

Thus we require

$$
a=\gamma^{2}\left(c^{2} B^{2} A^{3}\right), b_{1}=\gamma^{3}\left(c^{3} B^{4} A^{3}\right), \alpha b_{2}=\gamma^{3}\left(c^{3} B^{4} A^{3}\right), b_{0}=\alpha \gamma^{3}\left(c^{3} B^{2} A^{3}\right) .
$$

The first two relations give $\gamma=b_{1} /\left(a c B^{2}\right) \in H(4 d)$, so that also $\alpha \in H(4 d)$. The section $P$ on $X_{2}$ with $H(d)$-rational $y$-coordinate $y_{P}(t)$ pulls back to a section $P_{1}$ with $y^{\prime}$-coordinate $\gamma^{3 / 2} \alpha^{3} y_{P}(\alpha t)$. By construction, $P_{1}$ is $H(4 d)$-rational, so $\gamma^{3 / 2} \in H(4 d)$. But here $H(4 d)$ has degree three over $H(d)$, so $\gamma^{3 / 2} \in H(d)$. In other words, the isomorphism (15) is defined over $H(4 d)$.

In consequence, $Q$ pulls-back to a section on $X_{1}$ with $y^{\prime}$-coordinate $\sqrt{c_{Q}}$ times an $H(4 d)$-rational function. The same argument as for $\gamma^{3 / 2}$ then shows that $\sqrt{c_{Q}} \in$ $H(d)$. This gives the required contradiction.

4F. Proof of Theorem 2.4. We collect all results necessary to prove Theorem 2.4. Let $X$ be a singular K3 surface of discriminant $d$. We decided to work with Inose's pencil over $H(d)$ as in (14). Thus it suffices to check the field of definition of $\operatorname{MW}(X)$ to verify Theorem 2.4. In many cases, this was achieved in Lemma 3.4 
or in the intermediate Lemma 3.7 (as explained in Remark 3.8). For the remaining K3 surfaces, we considered the Kummer surface $X^{\prime}$ from the Shioda-Inose structure which actually sandwiches $X$ (4D). Note that for Kummer surfaces we exhibited a proof of Theorem 2.4 that only uses the techniques from Lemma 3.7 (Proposition 4.1, Corollary 4.2). Thanks to the interplay between $H(d)$ and $H(4 d)$, this suffices to deduce that $\mathrm{MW}(X)$ is defined over $H(d)$ by $4 \mathrm{E}$. This completes the proof of Theorem 2.4.

\section{Enriques surfaces of base change type}

This section provides a technique to construct explicit examples of Enriques surfaces whose covers are singular K3 surfaces. In the sequel, we refer to them as singular Enriques surfaces. The main idea is to invoke the base change construction from [Hulek and Schütt 2011, §3] for singular K3 surfaces. We will review the concept in 5B and then relate it to the Shioda-Inose structures from 3B.

5A. Singular K3 surfaces with Enriques involution. Our first problem concerns K3 surfaces: Which singular K3 surfaces admit an Enriques involution? Keum's result [1990] gives a partial answer for all singular K3 surfaces that are Kummer surfaces (i.e. with transcendental lattice two-divisible). The full problem can also be solved by purely lattice-theoretic means in terms of the transcendental lattice. In fact, one finds that the discriminant almost suffices to reach a decision: it suffices for non-Kummer surfaces while for Kummer surfaces we know the answer anyway from [Keum 1990]. Sertöz [2005] gave the solution based on the techniques developed by Keum:

Theorem 5.1. Let $X$ be a singular $K 3$ surface of discriminant $d$. Then $X$ does not admit an Enriques involution exactly in the following cases:

(i) $d \equiv-3 \bmod 8$,

(ii) $d=-4,-8$,

(iii) $d=-16$ and $X$ is not Kummer, i.e. $Q(X)=\operatorname{diag}(2,8)$.

Note that the discriminants in case (ii) determine unique singular K3 surfaces up to isomorphism. In case (iii), we have to exempt the Kummer surface $\operatorname{Km}\left(E_{i} \times E_{i}\right)$ with transcendental lattice of intersection form $Q=\operatorname{diag}(4,4)$ which admits an Enriques involution by [Keum 1990].

Sertöz's proof is purely lattice theoretic and based on machine computations. In particular, for those singular K3 surfaces admitting some Enriques involution, it does not give any explicit geometric description of any such involution. Here we shall combine the ideas from [Hulek and Schütt 2011, §3] and Section 3 to derive explicit Enriques involutions on almost all singular K3 surfaces possible according to Theorem 5.1. 
5B. Enriques involutions of base change type. We start by reviewing the set-up from [Hulek and Schütt 2011, §3]:

$S \quad$ rational elliptic surface

$f \quad$ quadratic base change of $\mathbb{P}^{1}$ (not ramified at nonreduced fibres of $S$ )

$X \quad$ base change of $S$ by $f:$ K3 surface

$l \quad$ base change involution

$(-1) \quad$ hyperelliptic involution

$\boxplus P \quad$ translation by a section $P \in \operatorname{MW}(X)$

In this situation, the composition $J=\imath \circ(-1)$ defines a Nikulin involution on $X$, i.e. $J$ has eight isolated fixed points and leaves the holomorphic two-form invariant. The quotient $X / \mathrm{J}$ has a resolution $X^{\prime}$ that is again $\mathrm{K} 3 . X^{\prime}$ is the quadratic twist of $S$ at the ramification points of the base change $f$ : The induced action of $l$ and $J$ gives a decomposition of $\mathrm{MW}(X)$ up to some 2-power index:

$$
\operatorname{MW}(X)_{\mathbb{Q}} \cong \operatorname{MW}(S)_{\mathbb{Q}}+\operatorname{MW}\left(X^{\prime}\right)_{\mathbb{Q}} .
$$

Let $P^{\prime} \in \mathrm{MW}\left(X^{\prime}\right)$ and $P$ denote the induced section on $X$. By construction, $P$ is anti-invariant for $\imath^{*}$. In consequence,

$$
\tau:=\boxplus P \circ \iota
$$

is an involution on $X$. By definition, this involution can only have fixed points on the fixed fibres of $\iota$. If these fibres are smooth, one has

$$
\operatorname{Fix}(\tau)=\varnothing \Longleftrightarrow P \cap O \cap \operatorname{Fix}(l)=\varnothing .
$$

The latter condition can be checked with $P^{\prime}$ on the ramified fibres of $X^{\prime}$ (generally of type $I_{0}^{*}$ ). Here $P^{\prime}$ has to meet nonidentity components.

Example 5.2. The prototype example for this construction is a two-torsion section $P$ induced from $X^{\prime}$ (or equivalently from $S$ since two-torsion is not affected by quadratic twisting). Outside characteristic two, such a section is always disjoint from $O$. For $\tau$ to have fixed points, one of the ramified fibres has to be singular such that it is additive or $P$ meets the identity component.

The latter occurs for Example 3.1: There is exactly one two-torsion section induced from $S$. This section $(t-1, t-1)$ meets both ramified fibres (at 0 and $\infty$ ) at their identity components. The other two-torsion sections are interchanged by $l$ (which is why (16) only holds after tensoring with $\mathbb{Q}$ ).

5C. We ask which singular $\mathrm{K} 3$ surfaces admit an Enriques involution of base change type. For now we only exclude 62 or 63 singular K3 surfaces as specified in Exception 5.5 (62 assuming some special cases of ERH; see 5E). 
Proposition 5.3. Let $X$ be a singular $K 3$ surface admitting an Enriques involution. Assume that $X$ is not among the 62 or $63 \mathrm{~K} 3$ surfaces from Exception 5.5. Then $X$ has an Enriques involution $\tau$ of base change type where the Nikulin quotient $X^{\prime}$ is a Kummer surface.

The proof of the proposition will be given in $5 \mathrm{E}$ and $5 \mathrm{~F}$. It is based on the Shioda-Inose structure of singular K3 surfaces to that we will return next.

One word about Exception 5.5: we do not believe this exception to be necessary, but we have not found a general argument to overcome it (cf. Remark 5.6). To illustrate this, we will show in $5 \mathrm{G}$ that Example 3.1 which falls under Exception 5.5 does indeed admit an Enriques involution of base change type (but we did not check whether the quotient $X^{\prime}$ is a Kummer surface).

5D. Enriques involutions and Shioda-Inose structures. Let $E, E^{\prime}$ denote elliptic curves and consider the corresponding Shioda-Inose structure as in 3B. Then $X^{\prime}=$ $\mathrm{Km}\left(E \times E^{\prime}\right)$ admits an Enriques involution by [Keum 1990], but how about the K3 surface $X$ from 3B that recovers the transcendental lattice of the abelian surface $E \times E^{\prime}$ ?

If $E$ and $E^{\prime}$ are not isogenous, then $X$ has Picard number $\rho(X)=18$ and the fibration (5) of Mordell-Weil rank zero yields

$$
\mathrm{NS}(X)=U+2 E_{8}(-1) .
$$

This lattice does not admit any primitive embedding of the Enriques lattice $U(2)+$ $E_{8}(-2)$ because of the 2-length. Hence the K3 surface $X$ cannot have an Enriques involution. We now consider the case where $E$ and $E^{\prime}$ are isogenous, possibly with CM.

Here is our main tool to construct explicit Enriques involutions: the ShiodaInose structure falls under the settings studied in 5B. We already chose the notation to indicate this: there is a $\mathrm{K} 3$ surface $X$ with a Nikulin involution yielding the Kummer surface $X^{\prime}$. Conversely, $X$ is obtained from $X^{\prime}$ by a quadratic base change. In terms of the elliptic fibration (5) on $X$, the Nikulin involution is given as

$$
J:(x, y, t) \mapsto\left(x / t^{4},-y / t^{6}, 1 / t\right) .
$$

Thus the quotient $X / \mathrm{J}$ attains singularities in the fibres at $t= \pm 1$ whose minimal resolution is $X^{\prime}$. In general, the quotient results in fibres of type $I_{0}^{*}$, but there are other possibilities as sketched in 3B. Concretely, there is another involution corresponding to the base change $\mathbb{P}^{1} \rightarrow \mathbb{P}^{1}$ induced by $X \rightarrow X^{\prime}$ :

$$
\imath=\jmath \circ(-1):(x, y, t) \mapsto\left(x / t^{4}, y / t^{6}, 1 / t\right) .
$$

The quotient $X / \imath$ gives a rational elliptic surface $S$. It extends the Shioda-Inose structure to the following diagram (where we could also add the induced elliptic 
fibrations):

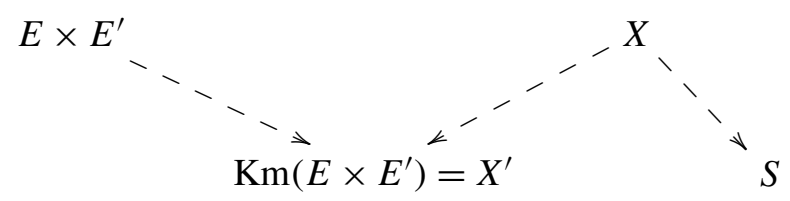

By construction, $S$ has a singular fibre of type $I I^{*}$. From the Shioda-Tate formula [Shioda 1990, Corollary 5.3], it follows that $S$ is extremal, i.e., it has finite MordellWeil group. Since a singular fibre of type $I I^{*}$ does not admit any torsion sections (of order relatively prime to the characteristic), we infer that $\mathrm{MW}(S)=\{O\}$. By [Shioda 1990, Proposition 8.12] (cf. (16)), this implies that

$$
\operatorname{MWL}(X)=\operatorname{MWL}\left(X^{\prime}\right)(2) \text {. }
$$

Hence as soon as the Mordell-Weil rank of $X$ is positive, there is a section $P$ (induced from $X^{\prime}$ ) and an involution $\tau$ as in 5B. In order to exhibit an Enriques involution on $X$, it remains to determine whether $\tau$ is fixed point free. In general there are three cases of positive Mordell-Weil rank to be distinguished according to the types of singular fibres. For nonsingular K3 surfaces, i.e., Mordell-Weil rank one with $E \not E^{\prime}$ and $\rho=19$, this has been done in [Hulek and Schütt 2011, $\S 4.2]$ (without referring to Shioda-Inose structures). The property whether $\tau$ is fixed point free or not depends on the parity of the height of the Mordell-Weil generator modulo 4 . In the next sections, we will treat the singular cases and thus prove Proposition 5.3.

Remark 5.4. There is a natural continuation of this connection between Enriques involutions of base change type and Shioda-Inose structures. Recall from Section 4 that the $\mathrm{K} 3$ surface $X$ is sandwiched by the Kummer surface $X^{\prime}$ in the following sense: $X^{\prime}$ can also be recovered from $X$ by the quadratic base change $u \mapsto t=u^{2}$ applied to (5). As in 5B, each section of $X$ induces an involution $\tau$ of base change type on the Kummer surface $X^{\prime}$. Here we ask whether $\tau$ is an Enriques involution. We have seen that the base change replaces the fibres of type $I I^{*}$ by type $I V^{*}$ (so these are fixed by $\tau$ ). However, none of these fibre types admits a free involution, so there cannot be an Enriques involution on $X^{\prime}$ as in 5B for the specified base change.

5E. Mordell-Weil rank one and $\boldsymbol{E} \cong \boldsymbol{E}^{\prime}$. In this case, $E$ is a CM elliptic curve with $j(E) \neq 0,12^{3}$. The elliptic fibration (5) on $X$ has exactly one reducible fibre of type $I_{2}$ at $t=1$ in addition to the two fibres of type $I I^{*}$. Together with the Mordell-Weil generator $P$, we can write

$$
\mathrm{NS}(X)=U+2 E_{8}(-1)+\left\langle A_{1}(-1), P\right\rangle .
$$


We consider two cases according to the intersection behaviour of the section $P$ and the fibre of type $I_{2}$.

If $P$ meets the nonidentity component of the $I_{2}$ fibre, then $P$ has height

$$
h(P)=4+2(P \cdot O)-1 / 2 .
$$

Equivalently, the discriminant $d=-2 h(P)$ of $X$ is odd. Clearly, $P$ and $O$ do not intersect on the $I_{2}$ fibre which is one of the two fixed fibres of the base change involution $l$. Here translation by $P$ exchanges the fibre components including the nodes, so it acts freely on the singular fibre. It remains to check for the specialisation of $P$ on the other fixed fibre at $t=-1$. Note that $P$ is induced from a section $P^{\prime}$ on the Nikulin quotient $X^{\prime}$, so

$$
P \cdot O=2\left(P^{\prime} \cdot O^{\prime}\right)+\# P \cap O \cap \operatorname{Fix}(l) .
$$

Since $P$ and $O$ can only possibly intersect on the irreducible fixed fibre of $l$ at $t=-1$, the parity of the intersection number $P \cdot O$ depends only the intersection behaviour at that fibre. In consequence, the discriminant $d$ of $X$ satisfies the congruence

$$
d \equiv-7 \bmod 8 \Longleftrightarrow P \cap O \cap \operatorname{Fix}(l)=\varnothing \Longleftrightarrow \operatorname{Fix}(\tau)=\varnothing .
$$

In comparison, Theorem 5.1 states that a singular K3 surface of odd discriminant $d$ admits an Enriques involution if and only if $d \equiv-7 \bmod 8$. This proves Proposition 5.3 for all odd discriminants and MW rank one cases. (As explained in $3 \mathrm{H}$ such fibrations exist on $X$ if and only if the transcendental lattice is primitive and lies in the principal genus.)

We now consider the case where $X$ has even discriminant, i.e., the section $P$ meets the identity component of the $I_{2}$ fibre. Then $\tau$ fixes both fibre components. As they are isomorphic to $\mathbb{P}^{1}$, there are fixed points. (In fact one can see that $\tau$ fixes one component pointwise.) In conclusion, the given elliptic fibration (5) on $X$ does not admit an Enriques involution of base change type.

This failure to produce an Enriques involution poses the problem how it can be overcome for the singular K3 surfaces in consideration for Proposition 5.3. Recall that we are in the special case where the fibration (5) corresponds to $E \cong E^{\prime}$. The principal idea now is to choose an alternative elliptic fibration of the same kind on $X$, but for a pair $\left(E, E^{\prime}\right)$ such that $E ¥ E^{\prime}$ (resembling our approach in $3 \mathrm{H}$ ). Whenever this is possible, the new fibration falls under the next case of MordellWeil rank two, and Proposition 5.3 can be proved along those lines. Here we can vary the pair $\left(E, E^{\prime}\right)$ by conjugates $\left(E^{\sigma},\left(E^{\prime}\right)^{\sigma^{-1}}\right)$. This fails to return a fibration of MW rank two if and only $E^{\sigma} \cong E^{\sigma^{-1}}$ for all Galois elements $\sigma$. Equivalently, the class group is only two-torsion. Note that $E \cong E^{\prime}$ implies that $T\left(E \times E^{\prime}\right)=T(X)$ is primitive and lies in the principal genus. Since the same applies to all conjugates, 
we derive the following abstract characterisation of the singular K3 surfaces where the Shioda-Inose structure does not produce an Enriques involution of base change type:

Exception 5.5. A singular K 3 surface $X$ of even discriminant $d$ does not admit an elliptic fibration (5) of Mordell-Weil rank two if and only if $T(X)$ is primitive and gives the full principal genus of its class group. In other words $Q(X)=$ $\operatorname{diag}(2,|d| / 2)$ and the class group $C l(d)$ is only two-torsion.

There are 101 known discriminants $d<0$ such that $C l(d)$ is only two-torsion; the discriminant of biggest absolute value is $d=-7392$. By [Weinberger 1973], there could be one more such discriminant of size $>10^{10}$, but this is ruled out by the extended Riemann hypothesis for odd real Dirichlet characters. Out of the 101 known discriminants, 65 are even (they were already studied by Euler, cf. [Cox 1989]) and $-4,-8,-16$ are ruled out by Theorem 5.1, so the above exception concerns 62 or 63 singular K3 surfaces. We consider one of them in detail in 5G after completing the proof of Proposition 5.3.

Remark 5.6. For each of the 62 known singular K3 surfaces from Exception 5.5, one could try to exhibit an Enriques involution as in 5B for a different base change than in the Shioda-Inose structure. However, there does not seem to be a universal way to achieve this. Notably, the general K3 surface $X$ arising from the ShiodaInose structure for the present case $E \cong E^{\prime}$ only admits four essentially different jacobian elliptic fibrations. To see this, one can argue with a gluing technique of Kneser-Witt that has been successfully applied to K3 surfaces in [Nishiyama 1996]. For these four fibrations, the fibre types reveal that only (5) and one other fibration can arise through a quadratic base change. The latter pulls back from the unique rational elliptic surface with a singular fibre of type $I_{9}$ and $\mathrm{MW}=\mathbb{Z} / 3 \mathbb{Z}$ by the one-dimensional family of quadratic base changes that ramify at the reducible fibre. A case-by-case analysis (exactly as above) shows that a singular elliptic K3 surface within this family can only have an Enriques involution of base change type if it does not fall under Exception 5.5.

5F. Mordell-Weil rank two. In this case, $E$ and $E^{\prime}$ are isogenous, but nonisomorphic elliptic curves with CM. Both fixed fibres for the base change involution $l$ at $t= \pm 1$ are smooth. On the Nikulin quotient $X^{\prime}$, they correspond to fibres of type $I_{0}^{*}$. As explained, the fibration (5) on $X$ has integral even Mordell-Weil lattice $\operatorname{MWL}(X)=\operatorname{MWL}\left(X^{\prime}\right)(2)=\operatorname{Hom}\left(E, E^{\prime}\right)(2)$, and

$$
\mathrm{NS}(X)=U+2 E_{8}(-1)+\operatorname{MWL}(X)(-1) .
$$

For an Enriques involution $\tau$ on $X$, we ask that some section $P \in \operatorname{MWL}(X)$ meets both fixed fibres at nonidentity components. Equivalently, there is a section $P^{\prime} \in$ 
$\operatorname{MWL}\left(X^{\prime}\right)$ (inducing $P$ ) that meets both ramified fibres (type $I_{0}^{*}$ ) at nonidentity components.

Assumption: There is no such section $P^{\prime} \in \operatorname{MWL}\left(X^{\prime}\right)$. Equivalently, since the simple components of a fibre admit a group structure, the nonidentity components of one of the $I_{0}^{*}$ fibres are fully avoided by $\mathrm{MW}\left(X^{\prime}\right)$. Correspondingly, $\mathrm{NS}\left(X^{\prime}\right)$ admits an orthogonal summand $D_{4}(-1)$ which we single out in the following decomposition:

$$
\mathrm{NS}\left(X^{\prime}\right)=U+E_{8}(-1)+D_{4}(-1)+\left\langle D_{4}(-1), \operatorname{MWL}\left(X^{\prime}\right)(-1)\right\rangle .
$$

Hence the discriminant group of $\mathrm{NS}\left(X^{\prime}\right)$ contains two copies of $\mathbb{Z} / 2 \mathbb{Z}$ (coming from $\left.D_{4}^{\vee} / D_{4}\right)$. Indeed, since the length is bounded by the rank of the transcendental lattice, which is two, this gives the full 2-part of the discriminant group:

$$
2-\operatorname{part}\left(\mathrm{NS}\left(X^{\prime}\right)^{\vee} / \mathrm{NS}\left(X^{\prime}\right)\right) \cong D_{4}^{\vee} / D_{4} \cong(\mathbb{Z} / 2 \mathbb{Z})^{2} \text {. }
$$

Right away, we deduce that $\mathrm{NS}\left(X^{\prime}\right)$ has discriminant $d^{\prime}$ equalling four times an odd integer. By (3), this odd integer is exactly the discriminant $d=d^{\prime} / 4$ of $X$. In particular, if $d$ is even, $\operatorname{MWL}\left(X^{\prime}\right)$ cannot fully avoid the nonidentity components of either of the $I_{0}^{*}$ fibres. Thus there is a section of the fibration (5) inducing an Enriques involution $\tau$ on $X$.

To complete the proof of Proposition 5.3, we return to the case of odd discriminant $d$. The isomorphism (17) gives an equality of discriminant forms

$$
-q_{D_{4}}=q_{D_{4}}=\left.\left(q_{\mathrm{NS}\left(X^{\prime}\right)}\right)\right|_{2 \text {-part }} .
$$

By [Nikulin 1980], there is an equality $q_{\mathrm{NS}\left(X^{\prime}\right)}=-q_{T\left(X^{\prime}\right)}$. Hence it suffices to compare the discriminant forms of $T\left(X^{\prime}\right)$ and $D_{4}$. In the present situation, $T\left(X^{\prime}\right)$ has the quadratic form

$$
\left(\begin{array}{ll}
4 a & 2 b \\
2 b & 4 c
\end{array}\right)
$$

with odd $b$. Hence its discriminant form takes the following values on a set of representatives of the 2-part of $T\left(X^{\prime}\right)^{\vee} / T\left(X^{\prime}\right)$ :

$$
0, a, c, a+b+c \quad \bmod 2 \mathbb{Z} \text {. }
$$

In comparison, $q_{D_{4}}$ does exclusively attain the value $1 \bmod 2 \mathbb{Z}$ on the nonzero elements of $D_{4}^{\vee} / D_{4}$. For $T\left(X^{\prime}\right)$, this can only happen if all $a, b, c$ are odd. Equivalently, the discriminant satisfies $d \equiv-3 \bmod 8$. This is exactly the main case excluded by Theorem 5.1.

Conversely, we deduce that a singular K3 surface $X$ admits an Enriques involution if it has an elliptic fibration (5) of Mordell-Weil rank two and if either $d$ is even or $d \equiv-7 \bmod 8$. The latter can be achieved unless $T(X)$ is primitive and 
corresponds to the principal class in its class group which is only two-torsion (cf. Exception 5.5). This completes the proof of Proposition 5.3.

5G. Appendix: More on Example 3.1. In this subsection, we will show that the singular K3 surface $X$ from Example 3.1 (which falls under Exception 5.5) does admit an alternative elliptic fibration with an Enriques involution of base change type. We will pursue an abstract approach following ideas of Kneser and Witt as worked out for elliptic K3 surfaces by Nishiyama [1996].

Lemma 5.7. $X$ has an elliptic fibration with $\mathbb{Z} / 3 \mathbb{Z} \subset \mathrm{MW}$ and two fibres of type $I_{9}$.

Proof. By [Nishiyama 1996, §6] the elliptic fibrations on $X$ are classified by primitive embeddings of a certain partner lattice $M$ of $T(X)$ into Niemeier lattices. Here we can take $M=A_{1}(-1)+A_{5}(-1)$ since $M$ and $T(X)$ have the same discriminant form. Consider the Niemeier lattice $N$ with root lattice

$$
N_{\text {root }}=A_{8}(-1)^{3} \text { and quotient } \quad N / N_{\text {root }}=(\mathbb{Z} / 3 \mathbb{Z})^{3} .
$$

Embedding $M$ primitively into one summand $A_{8}(-1)$, we obtain the essential lattice of an elliptic fibration of $X$ as orthogonal complement $M^{\perp} \subset N$. The singular fibres of this fibration are encoded in the roots of $M^{\perp}$, i.e., in $\left(M^{\perp}\right)_{\text {root }}=A_{8}(-1)^{2}$. The torsion in MW for this fibration is isomorphic to the quotient of the primitive closure of $\left(M^{\perp}\right)_{\text {root }}$ in $N$ by $\left(M^{\perp}\right)_{\text {root }}$, i.e., $\mathrm{MW}_{\text {tor }} \cong \mathbb{Z} / 3 \mathbb{Z}$.

The given elliptic fibration is not isotrivial due to the singular fibres of type $I_{9}$. The torsion in MW then implies that $X$ is a base change of the universal elliptic curve with 3-torsion section and j-invariant not identical zero. This elliptic surface has singular fibres $I_{1}, I_{3}, I V^{*}$, so necessarily the base change factors through the intermediate rational elliptic surface $S^{\prime}$ with configuration $I_{1}, I_{1}, I_{1}, I_{9}$ and $\mathrm{MW}\left(S^{\prime}\right)=\mathbb{Z} / 3 \mathbb{Z}$. In particular, $X$ arises from $S^{\prime}$ by a quadratic base change. Hence we are in the set-up of 5B with base change involution $\imath$ etc.

Now we consider the quadratic twist $X^{\prime}$. It is the desingularisation of the quotient of $X$ by the Nikulin involution $J=\imath \circ(-1)$. We claim that this quotient exhibits another Shioda-Inose structure on $X$ :

Lemma 5.8. $X^{\prime}$ is a Kummer surface with $T\left(X^{\prime}\right)=T(X)(2)$.

Proof. It suffices to prove that $J$ is a Morrison-Nikulin involution; i.e., $J^{*}$ exchanges two copies of $E_{8}(-1)$ in $\mathrm{NS}(X)$. Here we argue with the above elliptic fibration: $J$ exchanges the two reducible fibres of type $I_{9}$ and the three-torsion sections $Q, \boxminus Q$. Consider these 20 rational curves on $X$. Omitting the component of one $I_{9}$ fibre met by $Q$ and the component of the other $I_{9}$ fibre met by $\boxminus Q$, we find two disjoint configurations of type $\tilde{E}_{8}(-1)$ that are interchanged by $J$. The lemma now follows from [Morrison 1984, Theorem 5.7]. 
The induced elliptic fibration on $X^{\prime}$ has singular fibres $I_{1}, I_{1}, I_{1}, I_{9}, I_{0}^{*}, I_{0}^{*}$. Since $\rho(X)=20$, both $X$ and $X^{\prime}$ have MW-rank two. In particular, there are plenty of $\imath^{*}$-anti-invariant sections on $X$ (induced from $X^{\prime}$ ). As in 5B, each such section gives an involution $\tau$.

Lemma 5.9. There is a fixed-point free involution $\tau$ on $X$ as above.

Proof. We verify the claim on $X^{\prime}$ by assuming the contrary. This means that for one of the $I_{0}^{*}$ fibres all nonidentity components are avoided by $\mathrm{MW}\left(X^{\prime}\right)$. As in $5 \mathrm{~F}$, this implies that $X^{\prime}$ has discriminant four times an odd integer. But we have seen that $X^{\prime}$ has $T\left(X^{\prime}\right)=T(X)(2)$ with discriminant -48 . This gives a contradiction.

Remark 5.10. This example also shows that not every singular Enriques surface arises by the canonical Shioda-Inose structure from 5D. This fact can also be seen in terms of Enriques surfaces with finite automorphism group. Kondō classified these exceptional Enriques surfaces in [Kondō 1986]. Some are singular, but do not admit an elliptic fibration with a $I I^{*}$ fibre.

5H. Brauer groups. In [Hulek and Schütt 2011], we also answered a question by Beauville about Brauer groups. Namely Beauville asked for explicit examples of complex Enriques surfaces $Y$ where the $\operatorname{Brauer}$ group $\operatorname{Br}(Y) \cong \mathbb{Z} / 2 \mathbb{Z}$ pulls back identically zero to the covering K3 surface $X$ via the universal cover $\pi: X \rightarrow Y$. He also raised the question whether such an example exists over $\mathbb{Q}$.

In [Hulek and Schütt 2011, §5], we gave affirmative solutions for both questions. Our basic objects were the singular K3 surfaces $X$ with

$$
\mathrm{NS}(X)=U+2 E_{8}(-1)+\langle-4 M\rangle+\langle-2 N\rangle
$$

where $M, N \in \mathbb{N}$ and $N>1$ is odd. The above decomposition corresponds to an elliptic fibration (5) on $X$ with MW-rank two. As in 5B, the section $P$ of height $4 M$ induces an Enriques involution $\tau$ on $X$. Clearly the orthogonal section of height $2 N$ gives an anti-invariant divisor for $\tau^{*}$. By [Beauville 2009], this implies the vanishing of $\pi^{*} \operatorname{Br}(Y)$.

Previously we determined one surface (for $M=1, N=3$ ) with a model of (5) and Enriques involution $\tau$ defined over $\mathbb{Q}$. Here we want to point out that for any other surface $X$ as above, this can be achieved over the class field $H(-8 M N)$ by Theorem 1.1.

\section{Classification problems}

We conclude this paper by formulating classification problems for singular Enriques surfaces. In addition to fields of definition, we also consider Galois actions on divisors. First we review the situation for singular K3 surfaces. 
6A. Obstructions for singular K3 surfaces. Although singular K3 surfaces can often be descended from the ring class field $H(d)$ to some smaller number field, there are certain obstructions to this descent. In this section we shall discuss two of them. The first comes from the transcendental lattice. Since the Néron-Severi lattice of a general $\mathrm{K} 3$ surface is determined by intersection numbers, it is a geometric invariant; that is, conjugate surfaces have the same NS. Since $T(X)$ and NS $(X)$ are related as orthogonal complements in the K3 lattice $\Lambda$, they share the same discriminant form up to sign by [Nikulin 1980, Proposition 1.6.1]. In particular, this fixes the genus of $T(X)$ (sometimes also called the isogeny class).

Theorem 6.1 [Shimada 2009; Schütt 2007]. Let X be a singular K3 surface $X$ over some number field. The transcendental lattices of $X$ and its Galois conjugates cover the full genus of $T(X)$.

This result has an immediate consequence on the fields of definition:

Corollary 6.2. Let $X$ be a singular $K 3$ surface $X$ of discriminant $d$ over a number field L. Let $K=\mathbb{Q}(\sqrt{-d})$ and $\bar{L}$ the Galois closure of L over $K$. Denote by $G(X)$ the genus of $T(X)$. Then

$$
\# \mathscr{G}(X) \mid \operatorname{deg}_{K} L .
$$

In particular, one deduces that a singular K3 surface $X$ can only be defined over $\mathbb{Q}$ if the genus of $T(X)$ consists of a single class.

The second obstruction stems from the Galois action on the divisors. Namely, even if a singular K3 surface $X$ admits a model over a smaller field than $H(d)$, the ring class field is preserved through the Galois action on $\mathrm{NS}(X)$ :

Theorem 6.3 [Schütt 2010]. Let X be a singular K3 surface of discriminant d over some number field $L$. Assume that $\mathrm{NS}(X)$ is generated by divisors defined over $L$. Then the extension $L(\sqrt{d})$ contains the ring class field $H(d)$.

In other words, Theorem 2.4 is not far from being optimal: at best, there is a model with $\mathrm{NS}(X)$ defined over a quadratic subfield of $H(d)$.

Theorem 6.3 provides a direct proof of the following natural generalisation from CM elliptic curves, from [Shafarevich 1996]: Fixing $n \in \mathbb{N}$, there are only finitely many singular K3 surfaces over all number fields of degree bounded by $n$ (up to complex isomorphism). The problem of explicit classifications, however, is still wide open. Even in the simplest case, it is not clear yet how many singular K3 surfaces there are over $\mathbb{Q}$ - only that there are many, cf. [Elkies and Schütt $2008 \mathrm{~b}$ ]. In contrast, the restrictive setting of Theorem 6.3 is much more accessible. For instance there are exactly 13 singular K3 surfaces up to $\overline{\mathbb{Q}}$-isomorphism with NS defined over $\mathbb{Q}$. By [Schütt 2010, Theorem 1], they stand in bijective correspondence with the discriminants $d$ of class number one. 
We shall now discuss how these obstructions turn out for singular Enriques surfaces. Then we formulate analogous classification problems.

6B. Fields of definition of singular Enriques surfaces. We start by pointing out that Theorem 6.1 carries over to singular Enriques surfaces directly. This fact is due to the universal property that defines the covering $\mathrm{K} 3$ surface $X$ of an Enriques surface $Y$. Explicitly, $X$ can be defined universally as

$$
X=\operatorname{Spec}\left(\mathscr{O}_{Y} \oplus \mathscr{K}_{Y}\right)
$$

As this construction respects the base field, the obstructions from Theorem 6.1 on the field of definition of a singular K3 surface $X$ carry over to each singular Enriques surface that is covered by $X$. Recall that a K3 surface may admit (arbitrarily) finitely many distinct Enriques quotients by [Ohashi 2007, Theorem 0.1], while the universal cover associates a unique K3 surface to a given Enriques surface.

Corollary 6.4. Let $n \in \mathbb{N}$. There are only finitely many singular Enriques surfaces over all number fields of degree at most $n$ up to complex isomorphism.

Problem 6.5. The following two questions concern singular Enriques surfaces up to $\overline{\mathbb{Q}}$-isomorphism:

(1) For $n \in \mathbb{N}$, find all singular Enriques surfaces over number fields $L$ of degree at most $n$ over $\mathbb{Q}$.

(2) Specifically classify all singular Enriques surfaces over $\mathbb{Q}$.

6C. Galois action on divisors. Upon translating the obstructions for singular K3 surfaces from 6A to singular Enriques surfaces, we saw in 6B that Theorem 6.1 and its corollary carry over directly to the Enriques quotients. In contrast, Theorem 6.3 has to be weakened on the Enriques side. Generally speaking, this weakening is due to the fact that (part of) the Galois action can be accommodated by a sublattice of $\mathrm{NS}(X)$ that is killed by the Enriques involution. In support of these ideas, we shall review an example from [Hulek and Schütt 2011] (which draws heavily from [Elkies and Schütt 2008a]).

Consider the following family $\mathscr{L}$ of elliptic K3 surfaces

$$
\mathscr{X}: y^{2}=x^{3}+t^{2} x^{2}+t^{3}(t-a)^{2} x, \quad a \neq 0 .
$$

This elliptic fibration has reducible singular fibres of type $I I I^{*}$ at 0 and $\infty$ and $I_{4}$ at $t=a$. The general member has Picard number $\rho(\mathscr{X})=19$ with

$$
\operatorname{MW}(\mathscr{X})=\{O,(0,0)\} \cong \mathbb{Z} / 2 \mathbb{Z} .
$$


Note that $\mathscr{X}$ is of base change type - apply the base change $s=(t-a)^{2} / t$ to the rational elliptic surface $S$ with Weierstrass form

$$
S:=y^{2}=x^{3}+x^{2}+s x .
$$

As in 5B, the two-torsion section induces an Enriques involution $\tau$ (unless the other singular fibres degenerate, i.e., unless $a=-1 / 16$ ). Denote the family of Enriques quotients by 9 . We first study the Galois action on Num(Y):

Lemma 6.6. Let $Y_{a} \in \mathcal{Y}(a \neq-1 / 16)$. Then $\operatorname{Num}\left(Y_{a}\right)$ is defined over $\mathbb{Q}(a)$.

Proof. Since $\operatorname{Num}\left(Y_{a}\right)$ is torsion-free, the Galois action on $\operatorname{Num}\left(Y_{a}\right)$ coincides with that on the invariant part of $\operatorname{NS}\left(X_{a}\right)$. In the present situation, the $I_{4}$ fibre of $\mathscr{C}$ is split-multiplicative, i.e., all fibre components are defined over $\mathbb{Q}(a)$. The same holds trivially for the fibres of type $I I I^{*}$. Together with the sections $O$ and $(0,0)$, these rational curves generate $\operatorname{NS}\left(X_{a}\right)^{\tau^{*}}$ up to finite index. As this holds regardless of the Picard number of $X_{a}$ (being 19 or 20), the lemma follows.

Remark 6.7. It is crucial that the lemma holds for all members of the family 9 , including the singular ones. Compare the situation for singular K3 surfaces in the family $\mathscr{L}$ where Theorem 6.3 will often enforce a Galois action on the additional generator of NS. For the specialisations over $\mathbb{Q}$ with $\rho=20$, see $6 \mathrm{E}$.

6D. Néron-Severi group. We point out that in this specific setting, Lemma 6.6 gives a stronger statement than Corollary 2.5. The situation gets more complicated if we consider NS(Y) with its two-torsion because this can admit a quadratic Galois action. In particular, we can only conjecture an analogue of Corollary 2.5 for $\mathrm{NS}(Y)$ that is more precise than saying that $\mathrm{NS}(Y)$ is defined over some quadratic extension of $H(d)$ (Conjecture 6.11).

The main problem here lies in similar subtleties as encountered in the context of cohomologically and numerically trivial involutions (see [Hulek and Schütt 2011, $\S 4]$ and the references therein). Namely, to decide about $\mathrm{NS}(Y)$ it is necessary to work out generators of the full group (see Remark 6.9). We work this out for the family 9 in detail:

Proposition 6.8. If $Y_{a} \in \mathcal{Y}(a \neq-1 / 16)$, then $\operatorname{NS}\left(Y_{a}\right)$ is defined over $\mathbb{Q}(a, \sqrt{-a})$.

Proof. The next remark will indicate that it is not sufficient to argue with the elliptic fibration (19) on $\mathscr{X}$. Instead, we consider Inose's fibration (5) for the given family. The following Weierstrass form was derived in [Hulek and Schütt 2011, §5.3]:

$$
\mathscr{X}: y^{\prime 2}=x^{\prime 3}+(9 a-1) x^{\prime} / 9+\left(27\left(u-\frac{a^{3}}{u}\right)+81 a+2\right) / 27 .
$$


There is a section $P$ of height 4 (thus disjoint from the zero section) with $x^{\prime}$ coordinate

$$
P_{x^{\prime}}(u)=\left(3 u^{4}+12 u^{3} a+6 u^{2} a^{3}+4 u^{2} a^{2}-12 u a^{4}+3 a^{6}\right) /\left(12 a^{2} u^{2}\right) .
$$

The section $P$ is anti-invariant for the base change involution $\imath$ of the Shioda-Inose structure on $\mathscr{X}$ :

$$
\imath:\left(x^{\prime}, y^{\prime}, u\right) \mapsto\left(x^{\prime}, y^{\prime},-a^{3} / u\right)
$$

The base change involution composed with translation by $P$ defines an Enriques involution $\tau^{\prime}$ on $\mathscr{X}$ by $5 \mathrm{~B}$. Denote the family of Enriques quotients by $\mathscr{Y}^{\prime}$. By Kondō's classification in [Kondō 1986], 'Y' has finite automorphism group, and in particular $\tau$ and $\tau^{\prime}$ are conjugate in $\operatorname{Aut}(\mathscr{X})$ so that $\mathscr{Y} \cong \mathscr{Y}^{\prime}$.

We continue by determining an explicit basis of $\mathrm{NS}\left(\mathrm{Y}^{\prime}\right)$. The induced elliptic fibration on $y^{\prime}$ has a singular fibre of type $I I^{*}$, a bisection $R$ (the push-down of $O$ and $P$ ) and two multiple smooth fibres $F_{1}=2 G_{1}, F_{2}=2 G_{2}$. We claim that these twelve curves generate $\mathrm{NS}\left(\mathrm{Y}^{\prime}\right)$. To see this, note that by construction $R$ meets the simple component of the $I I^{*}$ fibre twice. The remaining fibre components form the root lattice of type $E_{8}(-1)$. Orthogonally in $\mathrm{NS}\left(\mathrm{Y}^{\prime}\right)$, we find $R, G_{1}, G_{2}$. Since $R^{2}=-2, R \cdot G_{i}=1$, we know that $R, G_{1}$ generate the hyperbolic plane $U$. Thus we have determined a unimodular lattice $L=U+E_{8}(-1)$ inside NS $\left(\mathscr{Y}^{\prime}\right)$ - necessarily of index two due to its rank being ten. Since $G_{2} \notin L$, it follows that $L$ and $G_{2}$ generate all of $\mathrm{NS}\left(\mathrm{Y}^{\prime}\right)$.

We now consider the Galois action on these generators of $\mathrm{NS}\left(Y_{a}^{\prime}\right)$ for some $Y_{a}^{\prime} \in \mathcal{Y}^{\prime}$. Clearly the $I I^{*}$ fibre and the bisection $R$ are defined over $\mathbb{Q}(a)$. The multiple fibres sit at the ramification points of the base change on the base curve $\mathbb{P}^{1}$, i.e., at the roots of $u^{2}+a^{3}$. Proposition 6.8 follows and cannot be improved since the conjugation of $\mathbb{Q}(\sqrt{-a}) / \mathbb{Q}(a)$ permutes the multiple fibres if $\sqrt{-a} \notin \mathbb{Q}(a)$, and thus gives a nontrivial Galois action on $\mathrm{NS}\left(Y_{a}^{\prime}\right)$.

Remark 6.9. Note that the above Galois action is not visible on the elliptic fibration (19) of $\mathscr{X}$ yielding $\mathscr{Y}$. The multiple fibres of the induced elliptic fibration on $y$ have different type $2 I_{0}, 2 I_{2}$. Hence they cannot be interchanged by Galois. Nonetheless there can be a nontrivial Galois action on $\operatorname{NS}\left(Y_{a}\right)$. This goes undetected in the above model because the push-down of fibre components and torsion sections from $\mathscr{X}$ to $\mathscr{Y}$ generate NS(Y) only up to index two.

6E. CM-points. Concretely, the family $\mathscr{X}$ is parametrised by the Fricke modular curve $X_{0}(2)^{+}$. In [Elkies and Schütt 2008a], we list all $\mathbb{Q}$-rational CM-points. Two of them give singular K3 surfaces without Enriques involution (discriminant -8 at $a=-1 / 16$ and discriminant -4 at $a=0$ for a suitable alternative model of $\mathscr{X}$ ). 
The other 14 discriminants are:

$$
-7,-12,-16,-20,-24,-28,-36,-40,-52,-72,-88,-100,-148,-232 .
$$

For the discriminants of class number two, the additional section can only be defined over a quadratic extension of $\mathbb{Q}$ by Theorem 6.3. So there are indeed singular Enriques surfaces with Num defined over $\mathbb{Q}$ where the same does not hold for the covering K3 surfaces. A detailed example where this holds even for NS is provided by the surfaces at $a=-1 / 144$ which corresponds to the discriminant -24 (as mentioned in 5H). Details can be found in [Hulek and Schütt 2011, §5.3]. We work out one example from the list where Num is defined over $\mathbb{Q}$, but NS is neither defined over $\mathbb{Q}$ nor over $H(d)$ :

Example 6.10. The specialisation $X$ with discriminant $d=-12$ sits at $a=1 / 9$. In terms of the elliptic fibration (19), there is a section of height 3 over $H(d)=$ $\mathbb{Q}(\sqrt{-3})$ with $x$-coordinate $-12 t^{3} /(9 t-1)^{2}$. One finds that $X$ has transcendental lattice two-divisible, so $X$ is the Kummer surface of $E \times E$ for $E$ with jinvariant zero. In particular $X$ is different from the singular $\mathrm{K} 3$ surface studied in Example 3.1 and 5G.

The Enriques quotient $Y$ has multiple fibres at $\pm \sqrt{-1} / 27$. Compared with $\operatorname{Num}(Y)$ which is defined over $\mathbb{Q}$, complex conjugation acts on $\mathrm{NS}(Y)$ as nontrivial Galois action. Note that $H(d)(\sqrt{-1})=H(4 d)$ in the present situation.

6F. In the above example (and in fact for all specialisations over $\mathbb{Q}$ with $\rho=20$ ), we have seen that $\mathrm{NS}(Y)$ is defined over the ring class field $H(4 d)$. We conjecture that this is always the case which would give an analogue of Corollary 2.5:

Conjecture 6.11. Let $Y$ be an Enriques surface whose universal cover $X$ is a singular K3 surface. Let $d<0$ denote the discriminant of $X$. Then $Y$ admits a model over the ring class field $H(d)$ with $\mathrm{NS}(Y)$ defined over $H(4 d)$.

The above one-dimensional family provides small evidence for this conjecture. Our main motivation stems from the base change construction of Enriques involutions in the framework of Shioda-Inose structures as investigated in Section 5. By Proposition 5.3, almost every possible singular K3 surface admits such an Enriques involution. In terms of the model (14), the Enriques quotient $Y$ attains multiple fibres at the ramification points of the underlying base change, i.e., at $\pm 2 B$. Recall from (5) that $B^{2}=\left(1-j / 12^{3}\right)\left(1-j^{\prime} / 12^{3}\right)$, so there is a quadratic Galois action on NS(Y) unless $B \in H(d)$. Note that $B$ can be interpreted in terms of the Weber function $\sqrt{j-12^{3}}$ where $j$ now denotes the usual modular function. The values of Weber functions at CM-point have been studied extensively starting from Weber. In the present situation, Schertz [1976] proved that for singular $j$-values, $\sqrt{j-12^{3}} \in H(4 d)$. This implies: 
Lemma 6.12 (Schertz). In the above setting, one has $B \in H(4 d)$.

We sketch an alternative proof of Lemma 6.12. It is based on a geometric approach that will also carry information about the Enriques surface $Y$ (and its elliptic fibration with fibre of type $I I^{*}$ ). Consider the Kummer surface $X^{\prime}$ from the Shioda-Inose structure. In general, it has fibres of type $I_{0}^{*}$ where $Y$ has the multiple fibres (if $E \cong E^{\prime}$, there could be fibres of type $I_{1}^{*}$ or $I V^{*}$; see $3 \mathrm{~B}$ ). By Corollary $4.2, X^{\prime}$ has a model with $\mathrm{NS}\left(X^{\prime}\right)$ defined over $H(4 d)$. In particular, every elliptic fibration of $X^{\prime}$ can be defined over $H(4 d)$ with all of NS defined there as well. We apply this argument to the elliptic fibration on $X^{\prime}$ induced from (14):

$$
X^{\prime}: y^{2}=x^{3}-3 c^{2} B^{2} A^{3}\left(t^{2}-4 B^{2}\right)^{2} x+c^{3} B^{2} A^{3}\left(t-2 B^{2}\right)\left(t^{2}-4 B^{2}\right)^{3} .
$$

Assume that $B \notin H(d)$ and denote $L=H(d)(B)$. By [Shioda 2006], the singular fibres of $X^{\prime}$ predict the Weierstrass form (20) (in case $A B \neq 0$ ) up to Möbius transformation. This property holds generally for constants $A, B, c$, but in the present situation, $A$ and $B$ are related to the j-invariants of $E, E^{\prime}$ by (5). Upon applying Möbius transformations, one can thus show that the above jacobian elliptic fibration does not admit a model over $H(d)$ without $\operatorname{Gal}(L / H(d))$-action interchanging the $I_{0}^{*}$ fibres. By Corollary 4.2, one obtains that $B \in H(4 d)$. This proves Lemma 6.12.

Corollary 6.13. Conjecture 6.11 holds true for any singular Enriques surface arising from the Shioda-Inose structure as in Section 5.

The geometric proof of Lemma 6.12 is of particular interest to us, since the statement about the Galois action on the $I_{0}^{*}$ fibres of $X^{\prime}$ carries over to the multiple fibres of the corresponding elliptic fibration of the Enriques surface $Y$ and vice versa. Centrally, we use once again that a model of a K3 or Enriques surface with NS defined over a fixed field has all elliptic fibrations (with or without section) defined over this field as well. Hence we can move freely between models and elliptic fibrations. Thus we obtain:

Corollary 6.14. If $B \notin H(d)$, then any model over $H(d)$ of the Enriques surface $Y$ admits a nontrivial Galois action of $\mathrm{Gal}(H(4 d) / H(d))$ on $\mathrm{NS}(Y)$.

We have seen an instance of this phenomenon in Example 6.10. The same reasoning implies a nontrivial action of $\operatorname{Gal}(\mathbb{Q}(a, \sqrt{-a}) / \mathbb{Q}(a))$ on $\mathrm{NS}\left(Y_{a}\right)$ for all $\mathbb{Q}(a)$-models of members $Y_{a}$ of the family $y$.

The above results allow us to draw an analogy to the study of automorphisms of Enriques surfaces; cf. [Barth and Peters 1983; Mukai and Namikawa 1984]. Namely we have exhibited two kind of singular Enriques surfaces over $H(d)-$ one with cohomologically trivial Galois action and one with numerically, but not cohomologically trivial Galois action. 
6G. We conclude this paper with the corresponding classification problem for singular Enriques surfaces. Note that by the above reasoning, at least the second problem is more complicated than for K3 surfaces (as solved in [Schütt 2010]).

Problem 6.15. The following two questions concern singular Enriques surfaces either up to $\overline{\mathbb{Q}}$ - or up to $L$-isomorphism:

(1) For a given number field $L$ (or all number fields of bounded degree), classify all singular Enriques surfaces with Num or NS defined over $L$.

(2) Determine all singular Enriques surfaces over $L=\mathbb{Q}$ with trivial Galois action on Num or NS.

\section{Acknowledgements}

We thank Bas Edixhoven and Jaap Top for useful comments. We are grateful to the referees for many helpful suggestions and remarks. This project was started when the second author held a position at University of Copenhagen.

\section{References}

[Barth and Peters 1983] W. Barth and C. Peters, "Automorphisms of Enriques surfaces", Invent. Math. 73:3 (1983), 383-411. MR 85g:14052 Zbl 0518.14023

[Barth et al. 2004] W. P. Barth, K. Hulek, C. A. M. Peters, and A. Van de Ven, Compact complex surfaces, 2nd ed., Ergebnisse der Mathematik (3) 4, Springer, Berlin, 2004. MR 2004m:14070 Zbl 1036.14016

[Beauville 2009] A. Beauville, "On the Brauer group of Enriques surfaces", Math. Res. Lett. 16:6 (2009), 927-934. MR 2011b:14079 Zbl 1195.14053

[Bogomolov and Tschinkel 1998] F. A. Bogomolov and Y. Tschinkel, "Density of rational points on Enriques surfaces”, Math. Res. Lett. 5:5 (1998), 623-628. MR 99m:14040 Zbl 0957.14016

[Cox 1989] D. A. Cox, Primes of the form $x^{2}+n y^{2}$ : Fermat, class field theory and complex multiplication, Wiley, New York, 1989. MR 90m:11016 Zbl 0701.11001

[Elkies and Schütt 2008a] N. D. Elkies and M. Schütt, "K3 families of high Picard rank", (2008).

[Elkies and Schütt 2008b] N. D. Elkies and M. Schütt, "Modular forms and K3 surfaces", 2008. arXiv math/0809.0830v2

[Gross 1980] B. H. Gross, Arithmetic on elliptic curves with complex multiplication, Lecture Notes in Mathematics 776, Springer, Berlin, 1980. MR 81f:10041 Zbl 0433.14032

[Hulek and Schütt 2011] K. Hulek and M. Schütt, "Enriques surfaces and Jacobian elliptic K3 surfaces”, Math. Z. 268:3-4 (2011), 1025-1056. MR 2818742 Zbl 1226.14052

[Inose 1978] H. Inose, "Defining equations of singular K3 surfaces and a notion of isogeny", pp. 495-502 in Proceedings of the International Symposium on Algebraic Geometry (Kyoto, 1977), edited by M. Nagata, Kinokuniya, Tokyo, 1978. MR 81h:14021 Zbl 0411.14009

[Keum 1990] J. H. Keum, "Every algebraic Kummer surface is the K3-cover of an Enriques surface", Nagoya Math. J. 118 (1990), 99-110. MR 91f:14036 Zbl 0699.14047

[Kondō 1986] S. Kondō, "Enriques surfaces with finite automorphism groups", Japan. J. Math. (N.S.) 12:2 (1986), 191-282. MR 89c:14058 Zbl 0616.14031 
[Morrison 1984] D. R. Morrison, "On K3 surfaces with large Picard number", Invent. Math. 75:1 (1984), 105-121. MR 85j:14071 Zbl 0509.14034

[Mukai and Namikawa 1984] S. Mukai and Y. Namikawa, "Automorphisms of Enriques surfaces which act trivially on the cohomology groups", Invent. Math. 77:3 (1984), 383-397. MR 86i:14012 Zbl 0559.14038

[Nikulin 1980] V. Nikulin, "Integral symmetric bilinear forms and some of their applications", Math. USSR, Izv. 14 (1980), 103-167. Zbl 427.10014

[Nishiyama 1996] K.-i. Nishiyama, "The Jacobian fibrations on some K3 surfaces and their MordellWeil groups”, Japan. J. Math. (N.S.) 22:2 (1996), 293-347. MR 97m:14037 Zbl 0889.14015

[Ohashi 2007] H. Ohashi, "On the number of Enriques quotients of a K3 surface", Publ. Res. Inst. Math. Sci. 43:1 (2007), 181-200. MR 2008b:14062 Zbl 1133.14038

[Piatetski-Shapiro and Shafarevich 1971] I. I. Piatetski-Shapiro and I. R. Shafarevich, "Torelli's theorem for algebraic surfaces of type K3", Izv. Akad. Nauk SSSR Ser. Mat. 35 (1971), 530-572. In Russian; translated in Math. USSR Izv. 5:3 (1971), 547-588. MR 44 \#1666 Zbl 0219.14021

[Schertz 1976] R. Schertz, "Die singulären Werte der Weberschen Funktionen $\mathfrak{f} \mathfrak{f}_{1}, \mathfrak{f}_{2}, \gamma_{2}, \gamma_{3}$ ”, $J$. Reine Angew. Math. 286/287 (1976), 46-74. MR 54 \#10205 Zbl 0335.12018

[Schütt 2007] M. Schütt, "Fields of definition of singular K3 surfaces", Commun. Number Theory Phys. 1:2 (2007), 307-321. MR 2008g:14060 Zbl 1157.14308

[Schütt 2008] M. Schütt, "Arithmetic of a singular K3 surface”, Michigan Math. J. 56:3 (2008), 513-527. MR 2009k:11106 Zbl 1163.14022

[Schütt 2010] M. Schütt, “K3 surfaces with Picard rank 20”, Algebra Number Theory 4:3 (2010), 335-356. MR 2011c:14113 Zbl 1190.14034

[Schütt and Shioda 2010] M. Schütt and T. Shioda, "Elliptic surfaces", pp. 51-160 in Algebraic geometry in East Asia (Seoul, 2008), edited by J. Keum et al., Adv. Stud. Pure Math. 60, Math. Soc. Japan, Tokyo, 2010. MR 2012b:14069 Zbl 1216.14036

[Sertöz 2005] A. S. Sertöz, "Which singular K3 surfaces cover an Enriques surface", Proc. Amer. Math. Soc. 133:1 (2005), 43-50. MR 2005m:14066 Zbl 1049.14032

[Shafarevich 1996] I. R. Shafarevich, "On the arithmetic of singular K3-surfaces”, pp. 103-108 in Algebra and analysis (Kazan, 1994), edited by M. M. Arslanov et al., de Gruyter, Berlin, 1996. MR 98h:14041 Zbl 0947.14020

[Shimada 2009] I. Shimada, "Transcendental lattices and supersingular reduction lattices of a singular K3 surface”, Trans. Amer. Math. Soc. 361 (2009), 909-949. MR 2009m:14055 Zbl 1187.14048

[Shimada and Zhang 2001] I. Shimada and D.-Q. Zhang, "Classification of extremal elliptic K3 surfaces and fundamental groups of open K3 surfaces", Nagoya Math. J. 161 (2001), 23-54. MR 2002d:14056 Zbl 1064.14503

[Shimura 1971] G. Shimura, Introduction to the arithmetic theory of automorphic functions, Publications of the Mathematical Society of Japan 11, Iwanami Shoten, Tokyo, 1971. MR 47 \#3318 Zbl 0221.10029

[Shioda 1990] T. Shioda, "On the Mordell-Weil lattices", Comment. Math. Univ. St. Paul. 39:2 (1990), 211-240. MR 91m:14056 Zbl 0725.14017

[Shioda 2006] T. Shioda, "Kummer sandwich theorem of certain elliptic K3 surfaces", Proc. Japan Acad. Ser. A Math. Sci. 82:8 (2006), 137-140. MR 2008b:14064 Zbl 1112.14044

[Shioda 2007] T. Shioda, "Correspondence of elliptic curves and Mordell-Weil lattices of certain elliptic K3's", pp. 319-339 in Algebraic cycles and motives, vol. 2, edited by J. Nagel and C. Peters, London Math. Soc. Lecture Note Ser. 344, Cambridge Univ. Press, 2007. MR 2009a:14052 Zbl 1136.14028 
[Shioda and Inose 1977] T. Shioda and H. Inose, "On singular K3 surfaces", pp. 119-136 in Complex analysis and algebraic geometry, edited by W. L. Baily, Jr. and T. Shioda, Iwanami Shoten, Tokyo, 1977. MR 56 \#371 Zbl 0374.14006

[Shioda and Mitani 1974] T. Shioda and N. Mitani, "Singular abelian surfaces and binary quadratic forms", pp. 259-287 in Classification of algebraic varieties and compact complex manifolds, edited by H. Popp, Lecture Notes in Mathematics 412, Springer, Berlin, 1974. MR 52 \#3174 Zbl 0302.14011

[Silverman 1994] J. H. Silverman, Advanced topics in the arithmetic of elliptic curves, Graduate Texts in Mathematics 151, Springer, New York, 1994. MR 96b:11074 Zbl 0911.14015

[Sterk 1985] H. Sterk, "Finiteness results for algebraic K3 surfaces", Math. Z. 189:4 (1985), 507513. MR 86j:14038 Zbl 0545.14032

[Tate 1975] J. Tate, "Algorithm for determining the type of a singular fiber in an elliptic pencil", pp. 33-52 in Modular functions of one variable, IV (Antwerp, 1972), edited by B. J. Birch and W. Kuyk, Lecture Notes in Math. 476, Springer, Berlin, 1975. MR 52 \#13850 Zbl 1214.14020

[Weinberger 1973] P. J. Weinberger, "Exponents of the class groups of complex quadratic fields", Acta Arith. 22 (1973), 117-124. MR 47 \#1776 Zbl 0217.04202

Communicated by János Kollár

Received 2010-03-13 Revised 2010-11-28 Accepted 2010-12-29

hulek@math.uni-hannover.de Institut für Algebraische Geometrie, Leibniz Universität Hannover, Welfengarten 1, 30167 Hannover, Germany

schuett@math.uni-hannover.de Institut für Algebraische Geometrie, Leibniz Universität Hannover, Welfengarten 1, 30167 Hannover, Germany 


\section{Algebra \& Number Theory}

msp.berkeley.edu/ant

\section{EDITORS}

MANAGING EDITOR

Bjorn Poonen

Massachusetts Institute of Technology

Cambridge, USA

\author{
EDITORIAL BOARD CHAIR \\ David Eisenbud \\ University of California \\ Berkeley, USA
}

\section{BOARD OF EDITORS}

Georgia Benkart

Dave Benson

Richard E. Borcherds

John H. Coates

J-L. Colliot-Thélène

Brian D. Conrad

Hélène Esnault

Hubert Flenner

Edward Frenkel

Andrew Granville

Joseph Gubeladze

Ehud Hrushovski

Craig Huneke

Mikhail Kapranov

Yujiro Kawamata

János Kollár

Yuri Manin

Barry Mazur

Philippe Michel

Susan Montgomery
University of Wisconsin, Madison, USA

University of Aberdeen, Scotland

University of California, Berkeley, USA

University of Cambridge, UK

CNRS, Université Paris-Sud, France

University of Michigan, USA

Universität Duisburg-Essen, Germany

Ruhr-Universität, Germany

University of California, Berkeley, USA

Université de Montréal, Canada

San Francisco State University, USA

Hebrew University, Israel

University of Kansas, USA

Yale University, USA

University of Tokyo, Japan

Princeton University, USA

Northwestern University, USA

Harvard University, USA

École Polytechnique Fédérale de Lausanne

University of Southern California, USA
Shigefumi Mori

Raman Parimala

Jonathan Pila

Victor Reiner

Karl Rubin

Peter Sarnak

Joseph H. Silverman

Michael Singer

Ronald Solomon

Vasudevan Srinivas

J. Toby Stafford

Bernd Sturmfels

Richard Taylor

Ravi Vakil

Michel van den Bergh

Marie-France Vignéras

Kei-Ichi Watanabe

Andrei Zelevinsky

Efim Zelmanov
RIMS, Kyoto University, Japan

Emory University, USA

University of Oxford, UK

University of Minnesota, USA

University of California, Irvine, USA

Princeton University, USA

Brown University, USA

North Carolina State University, USA

Ohio State University, USA

Tata Inst. of Fund. Research, India

University of Michigan, USA

University of California, Berkeley, USA

Harvard University, USA

Stanford University, USA

Hasselt University, Belgium

Université Paris VII, France

Nihon University, Japan

Northeastern University, USA

University of California, San Diego, USA

\section{PRODUCTION}

contact@msp.org

Silvio Levy, Scientific Editor

See inside back cover or www.jant.org for submission instructions.

The subscription price for 2012 is US \$175/year for the electronic version, and \$275/year (+\$40 shipping outside the US) for print and electronic. Subscriptions, requests for back issues from the last three years and changes of subscribers address should be sent to Mathematical Sciences Publishers, Department of Mathematics, University of California, Berkeley, CA 94720-3840, USA.

Algebra \& Number Theory (ISSN 1937-0652) at Mathematical Sciences Publishers, Department of Mathematics, University of California, Berkeley, CA 94720-3840 is published continuously online. Periodical rate postage paid at Berkeley, CA 94704, and additional mailing offices.

ANT peer review and production are managed by EditFLOW ${ }^{\circledR}$ from Mathematical Sciences Publishers.

PUBLISHED BY

mathematical sciences publishers

http://msp.org/

A NON-PROFIT CORPORATION

Typeset in IAT $_{\mathrm{E}} \mathrm{X}$

Copyright (C2012 by Mathematical Sciences Publishers 


\section{Algebra \& Number Theory}

Volume $6 \quad$ No. $2 \quad 2012$

Arithmetic of singular Enriques surfaces

Klaus HuleK and MatThias SchÜtT

An upper bound on the Abbes-Saito filtration for finite flat group schemes and applications

YICHAO TIAN

On the smallest number of generators and the probability of generating an algebra

Rostyslav V. Kravchenko, Marcin Mazur and Bogdan V. Petrenko

Moving lemma for additive higher Chow groups

AMALENDU KRISHNA and JINHYUN PARK

CHRISTOPHER GOFF

Uniformly rigid spaces

CHRISTIAN KAPPEN

On a conjecture of Kontsevich and Soibelman

LÊ QUY THUONG 